Louisiana State University

LSU Digital Commons

Faculty Publications

Department of Physics \& Astronomy

$12-2021$

\title{
Multi-scale feedback and feeding in the closest radio galaxy Centaurus A
}

\author{
B. McKinley \\ Curtin Univ, Int Ctr Radio Astron Res, Bentley, WA, Australia, ben.mckinley@curtin.edu.au
}

\section{S. J. Tingay}

Curtin Univ, Int Ctr Radio Astron Res, Bentley, WA, Australia

M. Gaspari

INAF, Osservatorio Astrofis \& Sci Spazio, Bologna, Italy; Princeton Univ, Dept Astrophys Sci, Princeton, NJ 08544 USA

R. P. Kraft

Harvard Smithsonian Ctr Astrophys, 60 Garden St, Cambridge, MA 02138 USA

C. Matherne

Louisiana State Univ, Dept Geol \& Geophys, Baton Rouge, LA 70803 USA

See next page for additional authors

Follow this and additional works at: https://digitalcommons.Isu.edu/physics_astronomy_pubs

Part of the Astrophysics and Astronomy Commons

\section{Recommended Citation}

McKinley, B., Tingay, S. J., Gaspari, M., Kraft, R. P., Matherne, C., Offringa, A. R., McDonald, M., Calzadilla, M. S., Veilleux, S., Shabala, S. S., Gwyn, S. D., Bland-Hawthorn, J., Crnojevic, D., Gaensler, B. M., \& JohnstonHollitt, M. (2021). Multi-scale feedback and feeding in the closest radio galaxy Centaurus A. NATURE ASTRONOMY https://doi.org/10.1038/s41550-021-01553-3

This Article is brought to you for free and open access by the Department of Physics \& Astronomy at LSU Digital Commons. It has been accepted for inclusion in Faculty Publications by an authorized administrator of LSU Digital Commons. For more information, please contact ir@lsu.edu. 


\section{Authors}

B. McKinley, S. J. Tingay, M. Gaspari, R. P. Kraft, C. Matherne, A. R. Offringa, M. McDonald, M. S. Calzadilla, S Veilleux, S. S. Shabala, S. D. J. Gwyn, J. Bland-Hawthorn, D. Crnojevic, B. M. Gaensler, and M. JohnstonHollitt 


\title{
Multi-scale feedback and feeding in the closest radio galaxy Centaurus $A$
}

\author{
B. McKinley $\oplus^{1 凶}$, S. J. Tingay', M. Gaspari $\oplus^{2,3}$, R. P. Kraft $\oplus^{4}$, C. Matherne ${ }^{5}$, A. R. Offringa ${ }^{6}$, \\ M. McDonald7, M. S. Calzadilla $\odot^{7}$, S. Veilleux ${ }^{8}$, S. S. Shabala ${ }^{9,10}$, S. D. J. Gwyn ${ }^{11}$, \\ J. Bland-Hawthorn $\left(^{12}\right.$, D. Crnojević ${ }^{13}$, B. M. Gaensler ${ }^{14}$ and M. Johnston-Hollittt ${ }^{15}$
}

\begin{abstract}
Supermassive black holes and supernova explosions at the centres of active galaxies power cycles of outflowing and inflowing gas that affect galactic evolution and the overall structure of the Universe $e^{1,2}$. While simulations and observations show that this must be the case, the range of physical scales (over ten orders of magnitude) and paucity of available tracers make both the simulation and observation of these effects difficult $t^{3,4}$. By serendipity, there lies an active galaxy, Centaurus A (NGC 5128) ${ }^{5,6}$, at such a close proximity as to allow its observation over this entire range of scales and across the entire electromagnetic spectrum. In the radio band, however, details on scales of 10-100 kpc from the supermassive black hole have so far been obscured by instrumental limitations ${ }^{7,8}$. Here we report low-frequency radio observations that overcome these limitations and show evidence for a broad, bipolar outflow with velocity of $1,100 \mathrm{~km} \mathrm{~s}^{-1}$ and mass-outflow rate of $2.9 \mathrm{M}_{\odot} \mathrm{yr}^{-1}$ on these scales. We combine our data with the plethora of multiscale, multi-wavelength, historical observations of Centaurus $A$ to probe a unified view of feeding and feedback, which we show to be consistent with the chaotic cold accretion self-regulation scenario $0^{9,10}$.
\end{abstract}

Centaurus A has been studied in great detail across the entire electromagnetic spectrum and has the largest angular extent of any radio galaxy, due to its close proximity 5 . The centre of the galaxy is just $3.8 \pm 0.1 \mathrm{Mpc}$ distant $^{11}$, such that $1 \mathrm{arcmin}$ on the sky equates to approximately $1 \mathrm{kpc}$. The radio source is characterized by a pair of bright inner lobes ${ }^{12}$, which extend approximately $5 \mathrm{kpc}$ from the nucleus and are being inflated by sub-parsec-scale radio jets (see Fig. 1 for the locations of these key features). The $8^{\circ}$ outer radio lobes span at least $480 \mathrm{kpc}$, with their true size probably larger since the northern jet points toward us and the southern counterpart away from $\mathrm{us}^{13,14}$, so the outer lobes probably also lie at an angle to our line-of-sight.

We used the Murchison Widefield Array ${ }^{15}$ (MWA) to observe the full extent of Centaurus A at a central frequency of $185 \mathrm{MHz}$ and with an angular resolution of $1.5 \mathrm{arcmin}$ (Methods), taking advantage of the MWA's extreme field-of-view, superb radio-quiet location and excellent sensitivity to large angular scales. The angular size and complexity of Centaurus A have long posed challenges for the imaging capabilities of radio telescopes. The MWA is able to meet these challenges, providing unique insights into the nature of this complex object. We detect previously unseen features throughout the diffuse outer lobes of the radio galaxy, which consist of a complex network of filaments (Fig. 1 and Extended Data Fig. 1). Of particular interest are the areas $\sim 1^{\circ}$ (tens of $\mathrm{kpc}$ ) north and south of the nucleus, which are transition regions through which energy must be transferred if feedback processes are able to substantially quench cooling flows, as proposed by theoretical models ${ }^{16,17}$.

With these transition regions revealed in our radio data, it is possible to study both the feeding and feedback processes occurring in Centaurus A, across the full range of physical scales and using a multi-wavelength approach. Considering the available suite of data on Centaurus A, we find that the unified model of active galactic nuclei (AGN) feeding and feedback ${ }^{9,17}$, based on chaotic cold accre$\operatorname{tion}^{10}(\mathrm{CCA})$ as the inflow mechanism, best explains the observations (see Methods for a detailed analysis and justification for the use of the unified CCA model). In this scenario, large-scale hot plasma halos cool and condense in a top-down multiphase cascade, forming clouds of cooler gas that rain down toward the nuclear region. A small amount of this matter is accreted, while most is ejected via fast outflows/jets that entrain further mass as they expand outwards with a larger opening angle. The model describes the link between the feeding of the AGN and the deposition of energy back into the surrounding medium via large-scale mechanical feedbacks and is agnostic to the source of the mechanical power. Wide, slow outflows at large radii are therefore characteristic of the model, but narrower radio jets, such as those seen directly in Centaurus A and other Fanarrof-Riley Type I sources, can still be present and contribute to a wide large-scale feedback process ${ }^{18}$.

The northern transition region (Fig. 1, top-right panel, and Fig. 2), is rich in features across the electromagnetic spectrum, including bright radio emission (commonly referred to as the northern middle lobe; $\left.\mathrm{NML}^{8,19}\right)$, X-ray knots $^{20}$, filaments of $\mathrm{H} \alpha$ and far-ultraviolet ${ }^{21}$ emission and clouds of $\mathrm{H} \mathrm{I}$ (ref. ${ }^{22}$ ) gas, as well as molecular gas and dust (ref. ${ }^{21}$ and references therein). These features indicate an environment with complex and ongoing interactions between gas of multiple phases and velocities, as predicted by the unified CCA model of AGN feedback and as seen as an emergent property of,

IInternational Centre for Radio Astronomy Research, Curtin University, Bentley, Western Australia, Australia. ${ }^{2}$ INAF, Osservatorio di Astrofisica e Scienza dello Spazio, Bologna, Italy. ${ }^{3}$ Department of Astrophysical Sciences, Princeton University, Princeton, NJ, USA. ${ }^{4}$ Harvard-Smithsonian Center for Astrophysics, Cambridge, MA, USA. ${ }^{5}$ Department of Geology \& Geophysics, Louisiana State University, Baton Rouge, LA, USA. ${ }^{6}$ Netherlands Institute for Radio Astronomy (ASTRON), Dwingeloo, the Netherlands. ${ }^{7}$ Kavli Institute for Astrophysics and Space Research, MIT, Cambridge, MA, USA. ${ }^{8}$ Department of Astronomy, Joint Space-Science Institute, University of Maryland, College Park, MD, USA. ' School of Natural Sciences, University of Tasmania, Hobart, Tasmania, Australia. ${ }^{10} \mathrm{ARC}$ Centre of Excellence for All Sky Astrophysics in 3 Dimensions (ASTRO-3D), Bentley, Western Australia, Australia. ${ }^{11}$ Canadian Astronomy Data Centre, Victoria, British Columbia, Canada. ${ }^{12}$ Sydney Institute for Astronomy, University of Sydney, Sydney, New South Wales, Australia. ${ }^{13}$ University of Tampa, Tampa, FL, USA. ${ }^{14}$ Dunlap Institute for Astronomy and Astrophysics, University of Toronto, Toronto, Ontario, Canada. ${ }^{15} \mathrm{Curtin}$ Institute for Computation, Curtin University, Perth, Western Australia, Australia. e-mail: ben.mckinley@curtin.edu.au 


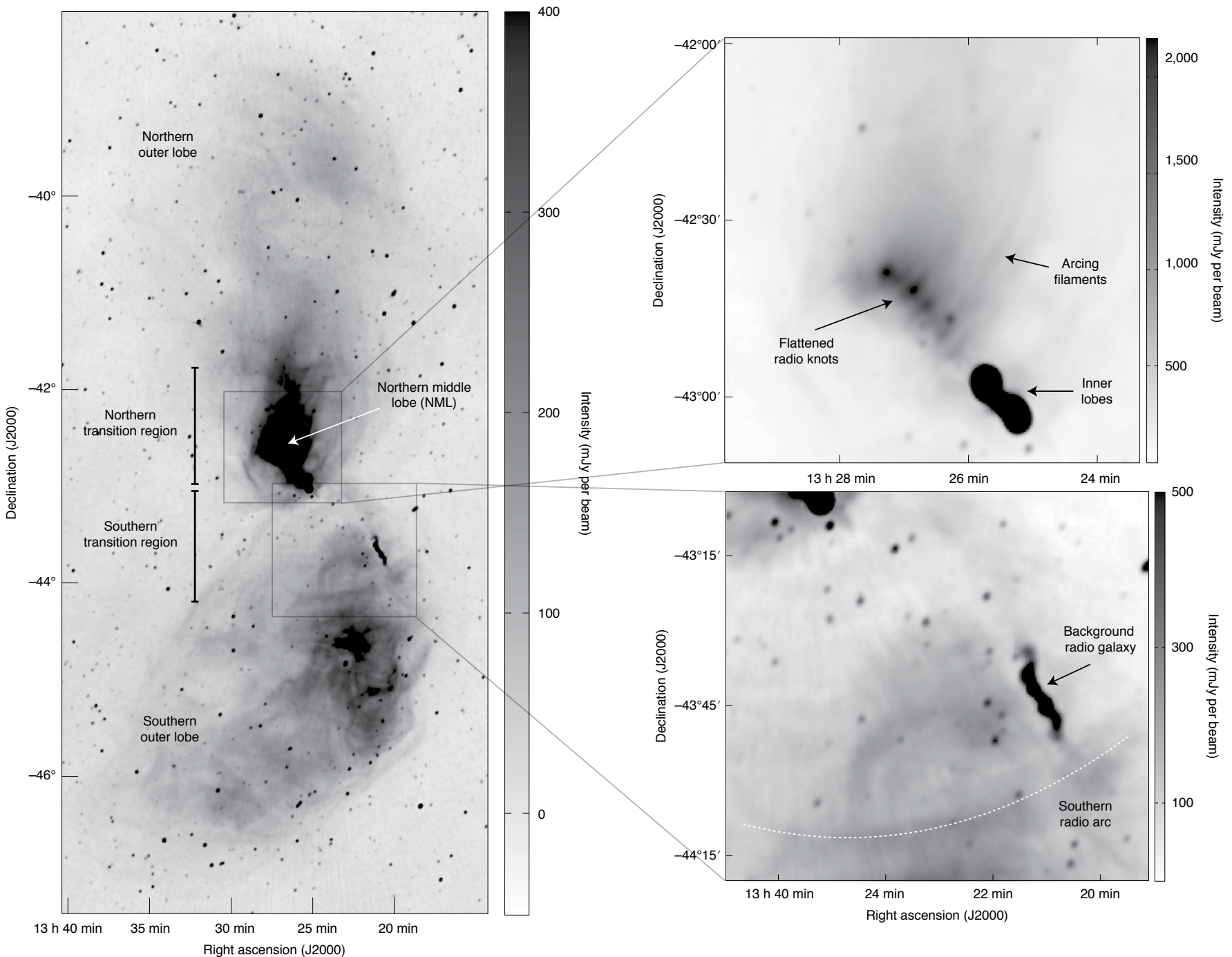

Fig. 1 | Our nearest neighbouring radio galaxy, Centaurus A, as seen at $185 \mathrm{MHz}$ with the Murchison Widefield Array. Left panel: the whole radio source shown on a linear intensity scale between -40 and 400 mJy per beam (the average restoring beam is a Gaussian of width $1.5 \times 1.2$ arcmin with a major axis position angle of $155^{\circ}$ ). Top-right panel: the northern transition region on a linear intensity scale of between -40 and 2,200 mJy per beam, showing the flattened radio knots and arcing filaments. Bottom-right panel: the southern transition region on a linear intensity scale of between 0 and $500 \mathrm{mJy}$ per beam, showing the southern radio arc. Each panel uses the same image data, and no post-processing image manipulation has been performed.

for example, the high-resolution Illustris 'The Next Generation' (TNG50) cosmological simulation ${ }^{3}$. Our radio image reveals details in this region (Fig. 1, top-right panel), not seen previously due to lack of sensitivity and/or imaging artefacts ${ }^{7,8,19}$. A series of bright radio knots arc across a path toward the northeast and are flattened along a northwest axis (this flattening was not apparent in the previous observations showing the knots ${ }^{8}$ ). Appearing to stream northwest from the flattened radio knots are faint, arcing filaments that follow the magnetic field direction ${ }^{23}$ in the region.

The leading scenarios for the origin of the X-ray and radio knots are that they are due to heated or multiphase gas clouds, or that they are star-formation sites ${ }^{8,21}$. The flattened nature of the radio knots strengthens the argument for a gas-cloud origin. In this scenario, the multi-wavelength features of the NML (Fig. 2) are being powered by a broad outflow from the AGN. The X-ray knots ${ }^{20}$ (red contours in Fig. 2) form where the mildly supersonic outflow impacts cold gas clouds in the region. The gas is compressed, bow shocks form upstream and the clouds' outer layers are heated to X-ray temperatures and ablated. This is seen in simulations of starburst winds impacting clouds of cold gas ${ }^{24,25}$. Radio knots, anti-coincident with the X-ray knots, form in front of the heated, X-ray emitting clouds, as electrons either encounter compressed magnetic fields and/or are accelerated at the bow shocks. An available source of gas to form the clouds is a partial ring of $\mathrm{H}_{\mathrm{I}}$ (Fig. 2, purple contour ${ }^{22}$ ), which is either the result of CCA processes or tidal disruption events. See Methods for a detailed discussion of the kinematics, energetics and timescales of these northern transition region gas clouds.

The arcing radio filaments replicate the shape and orientation of arcs of ionized gas observed on much smaller scales $(<1 \mathrm{kpc})$ in the outer ${ }^{26}$ and inner ${ }^{27,28}$ optical filaments. The arcing optical structures (Extended Data Fig. 2) bear a striking resemblance to features produced in simulations of broad transonic/supersonic winds ${ }^{25}$, or expanding jet cocoons ${ }^{28}$, impacting cold gas clouds. The matching shape and orientation of these optical arcs, and the radio filaments and flattened knots, indicates that they are being produced by the same outflow, which is much broader and far-reaching than what can be gleaned from observations of the optical filaments alone. Previous authors ${ }^{27}$ have suggested that the orientation of the inner filament arcs and velocity structure of the gas could be explained by the clouds being part of a backflow toward the AGN. Such inflowing 


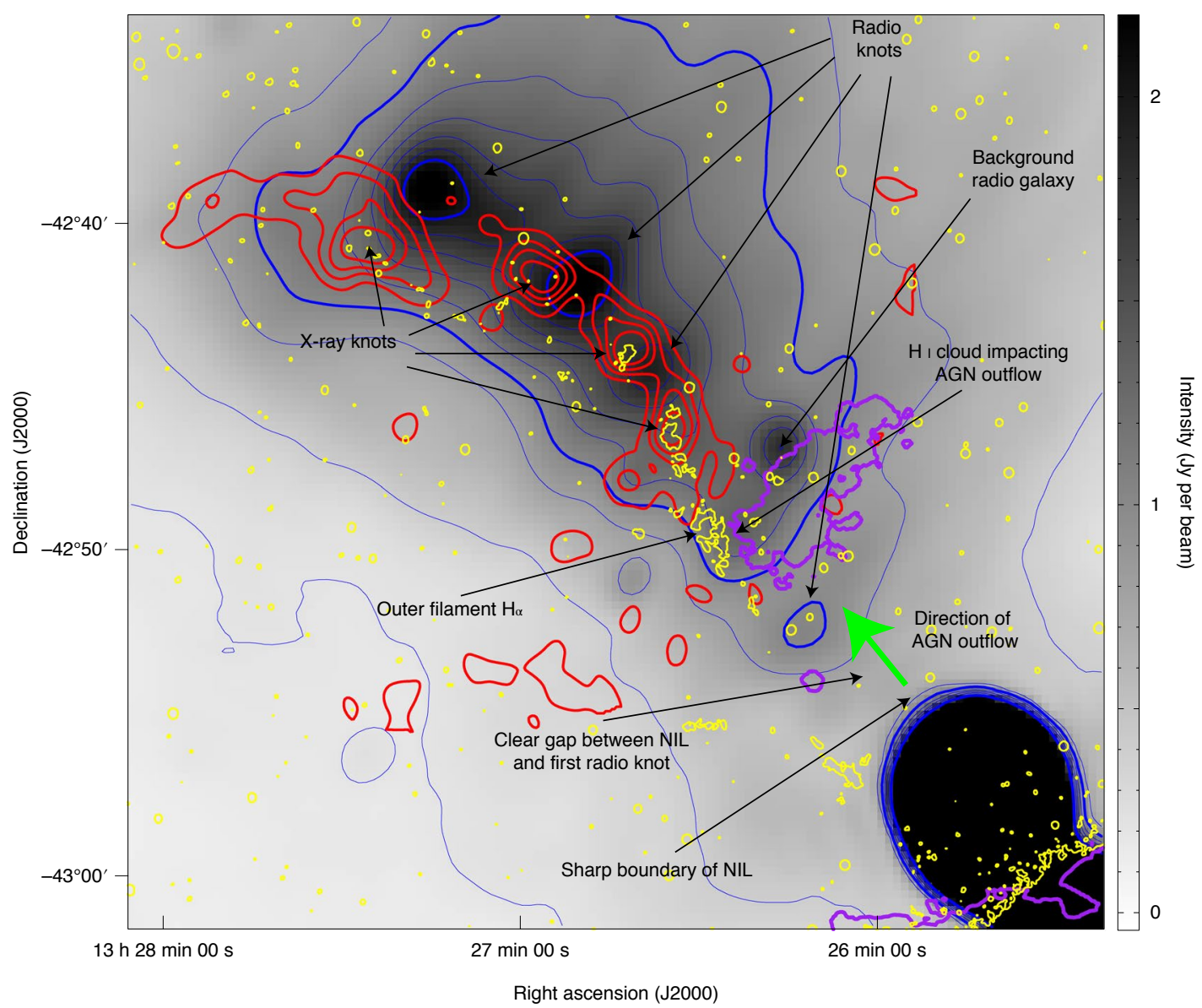

Fig. 2 | The northern transition region with multifrequency data overlaid. Contours are defined according to the s.d. ( $\sigma$ ) of a group of background pixels in the source image. Purple contour (at $5 \sigma$ above background), $\mathrm{H}$ I gas ${ }^{22}$; yellow contour (at $3 \sigma$ above background), H $\alpha$ emission (this work; Methods); red contours (at 2, 3, 4 and $5 \sigma$ above background), X-ray data ${ }^{20}$; blue contours (from 0.2 to 2.0 , incrementing by 0.2 Jy per beam) and greyscale, MWA $185 \mathrm{MHz}$ image (Fig. 1). Black regions are where the bright radio emission saturates the colour scale. The average restoring beam of the MWA image is a Gaussian of width $1.5 \times 1.2$ arcmin with a major axis position angle of $155^{\circ}$.

gas is predicted by the CCA model of self-regulated AGN feedback $^{9,17}$ and could exist alongside the outflow.

Another important radio feature in Fig. 2 is the sharp edge of the northern inner lobe (NIL) and a clear gap between it and the first flattened radio knot. This strongly disfavours the existence of a previously claimed ${ }^{19}$, highly collimated, 'large-scale jet' of relativistic material escaping from the NIL, confirming the interpretation of images of the NML at $327 \mathrm{MHz}$ (refs. ${ }^{8,21}$ ) that there is no tightly collimated supply of relativistic particles from the NIL to the NML.

Since we expect outflows from the AGN to be bipolar ${ }^{3}$, it is interesting to note the stark difference between the radio morphology of the northern and southern transition regions. As shown in Fig. 1, bottom-right panel, the region to the south of the inner lobes, down to a declination of around $-44^{\circ}$, displays none of the features seen to the north, except for a single semi-circular filament that lies just to the east of the bright, extended background radio galaxy MRC 1318-434B. Bordering this region, we highlight the 'southern radio arc'. This radio arc stands out for two main reasons: unlike other similar features in the image, it is roughly concentric with the active nucleus and it borders an area otherwise devoid of radio features (particularly if we consider that the smaller, closer arc may be in the foreground, appearing closer to the AGN only in projection). Given these distinctions, one plausible explanation for the southern radio arc is that it represents the zone where a broad transonic outflow, as identified to the north, transitions toward a strong interaction with the ambient medium in the south. The contrast in morphology to the north and south can be explained by differing environments, a scenario that is strengthened by considering the merger history of Centaurus A, which is traced by the presence of old, red-giant-branch (RGB) stars.

In Fig. 3, we reproduce a stellar-density map of RGB stars ${ }^{29}$ and mark the location of the $\sim 90$-kpc-long southern radio arc. There is a remarkable correspondence between the arc and the southern edge of a broad extension of RGB stars, which is not mirrored to the north of the galaxy. This stellar morphology can be largely accounted for by a major merger $\sim 2$ Gyr ago, as shown in simulated stellar-density maps of Centaurus $\mathrm{A}^{30}$. The additional tidal streams present in the actual data ${ }^{29}$ require infalling sub-structures at later times and in the northern region only. The 'second stream' identified by ref. ${ }^{29}$ (Fig. 3) is probably the remnant of a tidally disrupted dwarf, which has left behind dense gas in the northern transition region that may have disrupted the broad outflow in the north, while leaving the outflow unimpeded to the south. Such scenarios have been seen in simulations ${ }^{3}$, where both hierarchical structure formation and subsequent tidal disruption events produce asymmetric gaseous outflows similar to those observed in Centaurus A (for example, Fig. 2 of ref. $^{3}$ ).

With the above considerations, we contend that the southern radio arc approximately corresponds to the thermalization radius, defined as the radius where the pressure from a large-scale outflow equalizes with the hot-halo pressure ${ }^{17}$. Future studies, including a wider radio frequency range and X-ray data, are planned to test this interpretation. From Fig. 1, we estimate the thermalization radius as $75 \mathrm{kpc}$ and the opening solid angle of the outflow as 


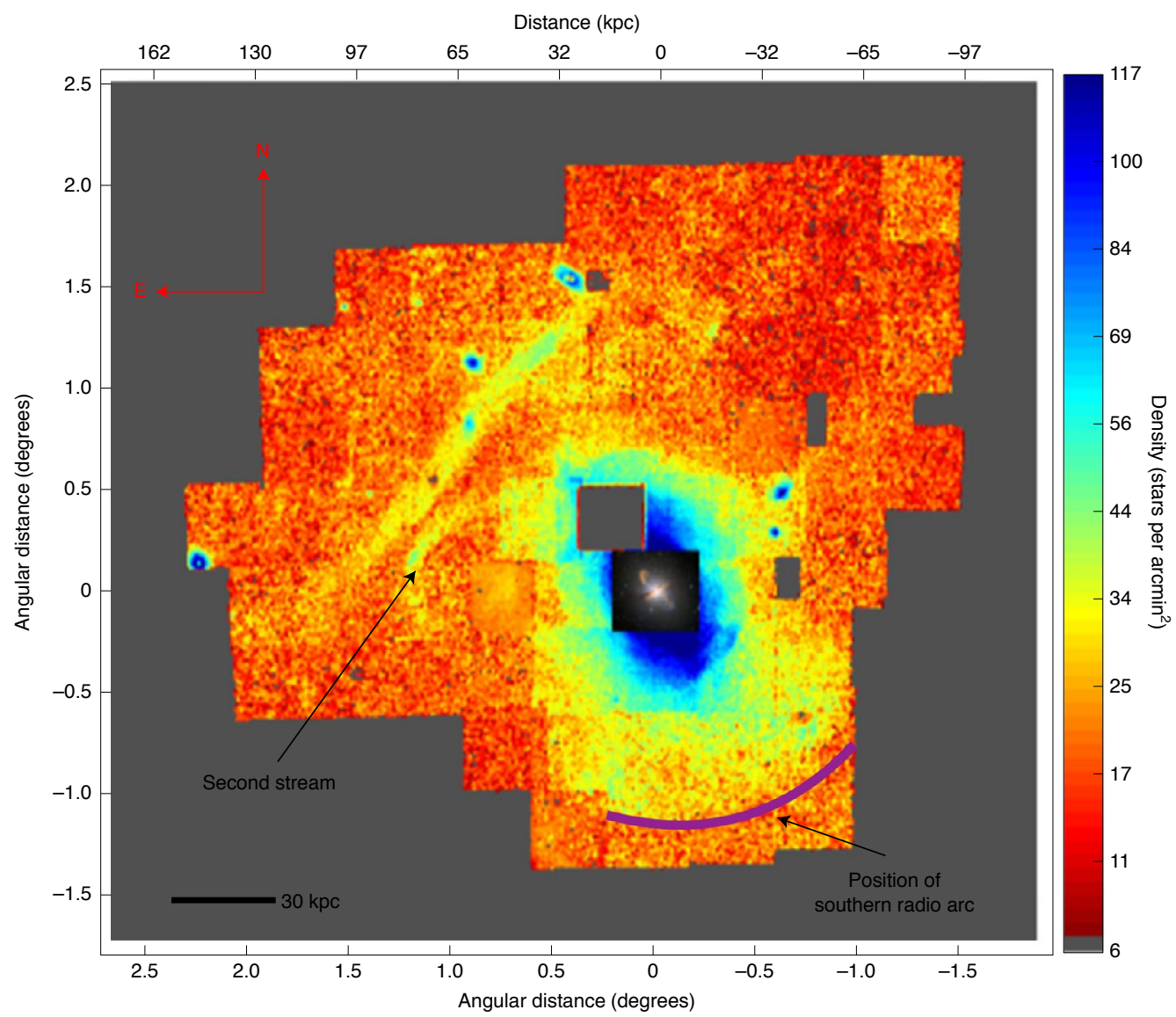

Fig. 3 Density map of RGB stars. Coordinates are centred on the core of Centaurus A and the position of the $90-\mathrm{kpc}$-long southern radio arc from Fig. 1 is shown as a magenta line at a radius of approximately $75 \mathrm{kpc}$. Figure reproduced with permission from ref. ${ }^{29}$, AAS.

$1.2 \mathrm{sr}$. We assume that the outflow is bipolar and that we do not see a corresponding similar, clear radio feature in the north, due to the gas asymmetries discussed earlier. Using these values and modified (to include the effect of a limited opening angle) versions of the equations describing the unified CCA model of AGN feedback ${ }^{17}$, we calculate the expected large-scale X-ray halo temperature and deduce a number of other AGN feedback properties for Centaurus A (see the Methods section for testing CCA, the full details of the related calculations and the comparisons with current Centaurus A observations via multiple scales and wavelengths). The inferred $\mathrm{X}$-ray halo temperature is $0.55 \mathrm{keV}$, giving a large-scale outflow speed of $\sim 1,100 \mathrm{~km} \mathrm{~s}^{-1}$ and a large-scale mass-outflow rate of $\sim 2.9 M_{\odot} \mathrm{yr}^{-1}$.

Having established the properties of the macro-scale outflow, we then calculate the corresponding feedback and feeding properties at small scales (Table 1). We infer a small-scale outflow speed of $\sim 7,100 \mathrm{~km} \mathrm{~s}^{-1}$, a mass-outflow rate of $\sim 0.07 M_{\odot} \mathrm{yr}^{-1}$ and a mass accretion rate onto the black hole (horizon) of $5.3 \times 10^{-4} M_{\odot} \mathrm{yr}^{-1}$. We refer to Methods for the full details of these calculations and a comprehensive comparison with current Centaurus A observations, covering an unprecedented range to link the macro, meso and micro scales (as suggested by the unification diagram in ref. ${ }^{9}$ ).

The feedback properties we derive are compatible with current Centaurus A data, consistent with relevant cosmological simulations and compatible with observations of AGN outflows at higher redshifts (Methods). Hence, we demonstrate that Centaurus A's proximity can be used to observe AGN outflows and inflows across ten orders of magnitude in physical scale and across a wide range of wavelengths. With the aid of high-resolution cosmological simulations, the insights gained from Centaurus A may be used in future work to better understand galaxies at higher redshifts. Overall, Centaurus A provides a key link between theory and observations by jointly probing multiple scales and phases, which will fundamentally advance our understanding of feedback and feeding throughout the local and distant Universe.

\section{Methods}

Radio observations and data processing. The $\mathrm{MWA}^{15,31}$ was used to produce the radio image shown in Fig. 1. The MWA is a low-frequency interferometer located at the radio-quiet future site of the low-frequency Square Kilometre Array ${ }^{32}$ in Western Australia. It has recently undergone an upgrade to phase 2, which has seen an increase in the maximum baseline length from 3 to $5.3 \mathrm{~km}$ (refs. ${ }^{33,34}$ ). Our image uses approximately 4 hours of data split evenly between phase 1 (with better low-surface-brightness sensitivity) and phase 2 (with better angular resolution), using the full MWA instantaneous bandwidth of $30.72 \mathrm{MHz}$ and a centre frequency of $185 \mathrm{MHz}$. Full details of the observations are given in Table 2. Particular care was taken to ensure the quality of data used. Radio frequency interference flagging was performed on the full-resolution data using AOFlagger ${ }^{35,36}$ during the standard pre-processing stages of the MWA pipeline and each measurement set was manually inspected using tools such as AOQuality and $\mathrm{AOQPlot}^{36}$. Bad antennas and baselines were manually flagged, and this inspection and flagging was repeated post-calibration.

Due to the complexity of Centaurus A and the lack of a detailed and accurate source model at low frequencies, we performed initial calibration using dedicated calibration scans of the bright radio source Pictor A. Phase and amplitude calibration were performed using Calibrate ${ }^{37}$ on a per-fine-channel basis, individually for each observation. Calibration solutions from the corresponding day (the phase-stability of the MWA allows us to use calibration solutions separated from target observations by several hours) were applied to target observations of Centaurus A. An initial set of 12 minutes of observation from both phase 1 and phase 2 (24 minutes in total) was then jointly imaged and deconvolved with WSClean ${ }^{38}$, using an implementation of the image domain gridding ${ }^{39}$ algorithm. The implementation of image domain gridding, along with integration of the MWA primary beam pattern ${ }^{40}$, allows accurate joint deconvolution of multiple MWA observations with different pointing directions (and hence different 
Table 1 | Unified AGN feedback properties of Centaurus A

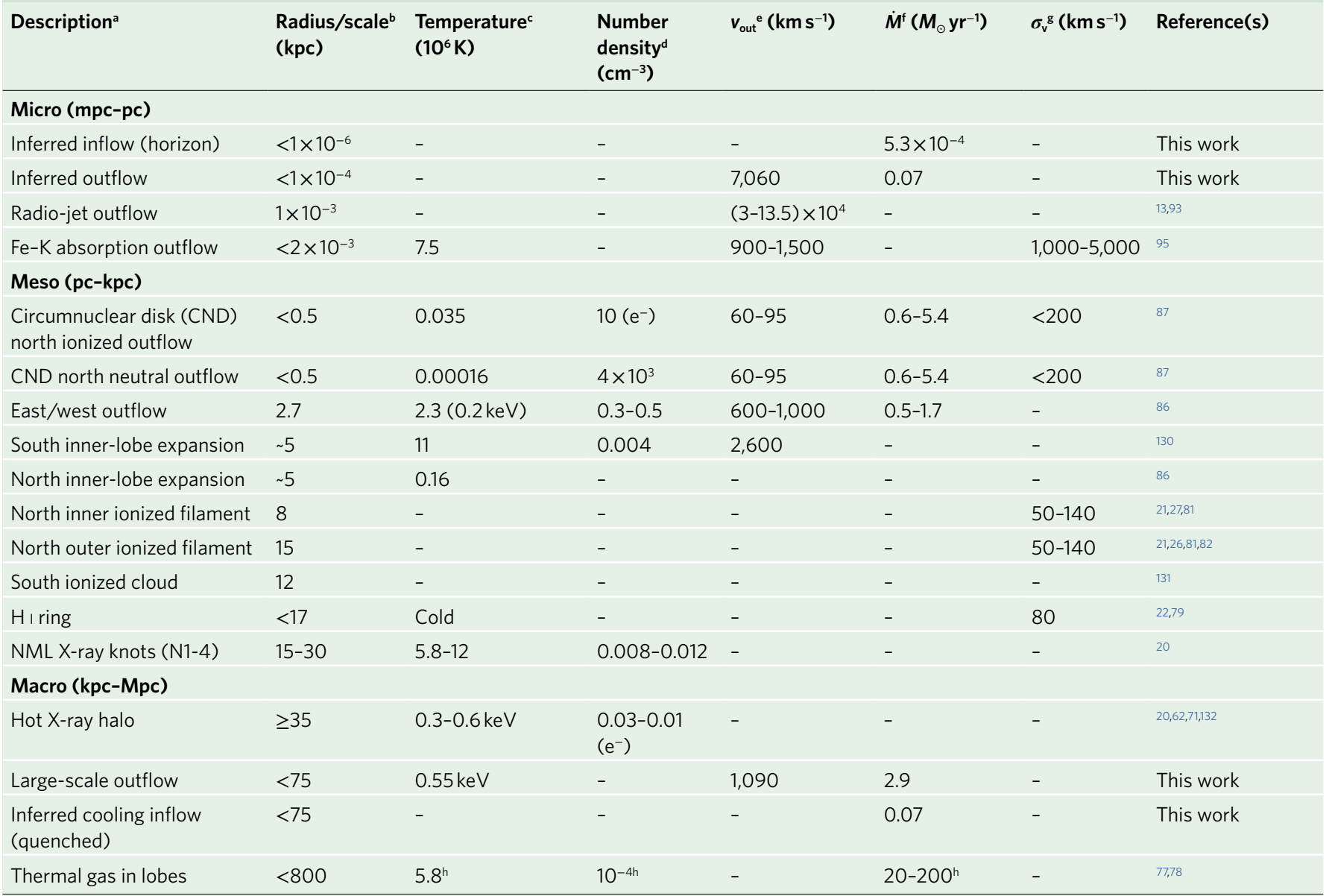

aRelevant AGN feedback feature, for example, inflow or outflow, gas cloud/knot/filament. ${ }^{\mathrm{b}} \mathrm{Approximate}$ radius from the nucleus. ${ }^{\mathrm{C}}$ Temperature of the gas in units of $10^{6} \mathrm{~K}$, or keV when more relevant. ${ }^{\mathrm{d} N u m b e r}$ density of gas; this is proton density unless labelled $\mathrm{e}^{-}$for electron density. ${ }^{\circ} \mathrm{Outflow}$ velocity. ${ }^{Mass}$ outflow rate. ${ }^{\mathrm{g}}$ Velocity dispersion. ${ }^{\mathrm{h}}$ These values may be excessively high due to Galactic foreground contamination; see discussion in Methods.

primary beam shapes). The limit of 24 minutes of observation was due to memory and CPU time considerations.

The resulting image from 24 minutes of observation was limited in quality in the outer regions, due to radial artefacts emanating from the bright inner lobes. However, the model produced by WSClean of the inner region, including the inner lobes and the NML, was of sufficient fidelity to be used as the calibration model for the remaining observations. Hence, another seven images were produced from $\sim 24$ minutes of observation each, with a direct source-model calibration and joint deconvolution. The image quality of each of these eight individual images varied, probably due to differing ionospheric conditions during the observations, with the main limiting factor being the radial artefacts similar to those seen in the initial image. Due to the different interferometric baseline coordinate $(u, v)$ coverages of the data, the radial lines were attenuated when the eight images were averaged together. We used a simple weighting scheme based on the root-mean-square (r.m.s.) noise in a region to the southwest of the AGN to form a weighted average of the eight images, which is the final product shown in Fig. 1. Details, including the Gaussian restoring beams and r.m.s. noise levels of the individual images and the final image, are shown in Table 2.

The final image has an angular resolution characterized by an average Gaussian restoring beam of width $1.5 \times 1.2 \mathrm{arcmin}$ with a major axis position angle of $155^{\circ}$, a r.m.s. background noise level of approximately $4 \mathrm{mJy}$ per beam and a peak brightness of 202 Jy per beam (giving a dynamic range of $\sim 50,000$ ). It is the most detailed and accurate image of the whole radio source so far, particularly within $1^{\circ}$ of the inner lobes, where previous MWA ${ }^{41}$ and Australia Telescope Compact Array /Parkes ${ }^{7}$ images suffered from artefacts that obscured the details of the north and south transition regions (this region was also not covered in the spectacular polarization images of the southern outer lobe from the Australian Square Kilometre Array Pathfinder ${ }^{42}$, for the same reason). The radio galaxy spans approximately $8^{\circ}$ on the sky along its north-south axis (the largest angular extent of any radio galaxy by a factor of 8 ) but is captured entirely within the wide field-of-view of the MWA, eliminating any need for mosaicing. Crucially, and unlike previous images of Centaurus $\mathrm{A}^{7,41}$, the image is largely free from artefacts, with the exception of some faint remaining radial striping that does not affect our analysis. Within the inner $\sim 1^{\circ}$ radius of the AGN, we achieve a factor of $\sim 500$ improvement in dynamic range compared with the next best image of the entire source with a similar angular resolution?

Previous images covering just the NML region of Centaurus A have also suffered from extensive imaging artefacts. The 'large-scale jet' identified in Australia Telescope Compact Array images ${ }^{19}$ at $1.4 \mathrm{GHz}$ and used to justify many theories of the NML's origin over the past $\sim 20$ years is not present in our radio image, nor the relatively recent Very Large Array (VLA) observations of the NML at $327 \mathrm{MHz}$ $\left(\right.$ ref. $\left.^{8}\right)$, indicating that it is in fact an imaging artefact. While the VLA observations failed to detect the large-scale jet, they were also hampered by severe image distortions due to the low elevation of Centaurus A when viewed from the VLA site.

To deal with the extreme dynamic range (which results in saturation of the central $\sim 1^{\circ}$ of Fig. 1, left panel) and to show all the radio features in a single image, the intensity scale was manipulated to produce Extended Data Fig. 1. In the right panel of Extended Data Fig. 1 the image has been stretched using the MaskedStretch tool of the astrophotography programme PixInsight (https:// pixinsight.com) and denoised to reduce the faint radial striping from the core. The MaskedStretch algorithm brings out all the features present in the image over a very large dynamic range by iteratively performing a series of weak nonlinear stretches, while masking the image with the result of the previous iteration at each step, so that high values are not clipped. A mean target background value of $0.05 \mathrm{Jy}$ per beam was used and the programme ran for 100 iterations. In the left panel of Extended Data Fig. 1, colour has been added using Photoshop. Yellow and white indicate higher intensities, while red and magenta represent lower intensities. The post-processing image manipulations used in Extended Data Fig. 1 produce a striking pair of images that display the key features of the radio galaxy, however their nonlinear nature renders the images unsuitable for quantitative analyses.

Optical observations and data processing. The yellow $\mathrm{H} \alpha$ contour in Fig. 2 is from wide-field optical data obtained using an astrophotography setup between June 2019 and February 2020. The observations were made from Río Hurtado, 
Table 2 | Details of MWA radio observations

\begin{tabular}{|c|c|c|c|c|c|c|c|}
\hline $\begin{array}{l}\text { Image } \\
\text { group }\end{array}$ & Phase 1 observation IDs & $\begin{array}{l}\text { Phase } 1 \\
\text { dates }\end{array}$ & Phase 2 observation IDs & Phase 2 dates & $\begin{array}{l}\text { Integration } \\
\text { time (min) }\end{array}$ & $\begin{array}{l}\text { Gaussian restoring } \\
\text { beam parameters: } \\
\text { major axis width, } \\
\text { minor axis width, } \\
\text { position angle } \\
\text { (arcmin, arcmin, } \\
\text { degrees from north } \\
\text { through east) }\end{array}$ & $\begin{array}{l}\text { r.m.s. } \\
\text { noise } \\
\text { (mJy per } \\
\text { beam) }\end{array}$ \\
\hline \multirow[t]{3}{*}{$\begin{array}{l}\text { Cen A } \\
\text { image } 1\end{array}$} & 1117031728,1121334536 & $\begin{array}{l}\text { May-July } \\
2015\end{array}$ & 1199663088,1200604688 & January 2018 & 33.1 & $1.37,1.12,155.0$ & 10.80 \\
\hline & 1121507392,1121593824 & & 1200777584,1200864032 & & & & \\
\hline & 1121420968,1121680256 & & 1200691136,1200950480 & & & & \\
\hline \multirow{3}{*}{$\begin{array}{l}\text { Cen A } \\
\text { image. } 2\end{array}$} & 1122198832,1122112400 & July 2015 & 1201123376,1201296272 & February 2018 & 35.2 & $1.48,1.23,163.0$ & 15.90 \\
\hline & 1121939544,1121853112 & & 1201469160,1201555608 & & & & \\
\hline & 1122025976,1121766680 & & 1201382720,1201814952 & & & & \\
\hline \multirow[t]{3}{*}{$\begin{array}{l}\text { Cen A } \\
\text { image } 3\end{array}$} & 1122285264,1122371696 & $\begin{array}{l}\text { July-August } \\
2015\end{array}$ & 1201895248,1201900584 & February 2018 & 31.5 & $1.51,1.29,163.4$ & 12.30 \\
\hline & 1122544552,1122630984 & & 1201981408,1201986752 & & & & \\
\hline & 1122458120,1122717416 & & 1201901392,1201987840 & & & & \\
\hline \multirow[t]{3}{*}{$\begin{array}{l}\text { Cen A } \\
\text { image } 4\end{array}$} & 1112806040,1112892200 & $\begin{array}{l}\text { April-May } \\
2015\end{array}$ & 1202239904,1202326064 & February 2018 & 23.2 & $1.40,1.06,145.1$ & 9.50 \\
\hline & 1114869144,1114955312 & & 1202411608,1202418952 & & & & \\
\hline & 1114782984,1115041472 & & 1202410528,1202672864 & & & & \\
\hline \multirow{3}{*}{$\begin{array}{l}\text { Cen A } \\
\text { image } 5\end{array}$} & 1115049272,1115127640 & May 2015 & 1202673200,1202673440 & February 2018 & 23.2 & $1.44,1.15,158.9$ & 9.85 \\
\hline & 1115213800,1115221600 & & 1202673920,1202674160 & & & & \\
\hline & 1115135440,1115299968 & & 1202673680,1202678472 & & & & \\
\hline \multirow{3}{*}{$\begin{array}{l}\text { Cen } A \\
\text { image } 6\end{array}$} & 1115307768,1116343632 & May 2015 & 1202679384,1202756888 & February 2018 & 24.1 & $1.37,1.16,159.9$ & 17.40 \\
\hline & 1116603776,1116604056 & & 1202843048,1202851152 & & & & \\
\hline & 1116429792,1116773232 & & 1202764984,1203015384 & & & & \\
\hline \multirow{3}{*}{$\begin{array}{l}\text { Cen A } \\
\text { image } 7\end{array}$} & 1120470256,1120815968 & July 2015 & 1242739136,1243169056 & May-June 2019 & 28.8 & $1.58,1.21,147.3$ & 9.28 \\
\hline & 1120988824,1121075248 & & 1243859272,1244376256 & & & & \\
\hline & 1120902392,1121161680 & & 1243341384,1244807072 & & & & \\
\hline \multirow[t]{3}{*}{$\begin{array}{l}\text { Cen A } \\
\text { image } 8\end{array}$} & 1121248104,1121334536 & July 2015 & 1234292944,1236791704 & $\begin{array}{l}\text { February-May } \\
2019\end{array}$ & 28.8 & $1.65,1.33,145.3$ & 8.45 \\
\hline & 1121507392,1121593824 & & 1242049824,1242221248 & & & & \\
\hline & 1121420968,1121680256 & & 1241705168,1242479744 & & & & \\
\hline Final image & & & & & 3.8 hours & $1.5,1.2,155$ & 4.00 \\
\hline
\end{tabular}

Chile, utilizing a Takahashi TOA-150 refracting telescope and Finger Lakes Instrumentation ML 16200 monochrome charge-coupled device operated at $-25^{\circ} \mathrm{C}$, onboard an Astro-Physics AP 1600 mount. The light was filtered using a set of luminance (L), red (R), green (G), blue (B) and $8 \mathrm{~nm} \mathrm{H} \alpha$ filters from Finger Lakes Instrumentation, with total exposure times of $13.25,7,7.5,7.25$ and 32.5 hours, respectively, giving a combined exposure time for the RGB image of 67.5 hours. The light frames were calibrated using 30 dark frames, 30 flat frames per filter and 50 bias frames taken under identical conditions as the light frames, before being aligned and integrated using the winsorized sigma rejection algorithm in PixInsight. Figure 2 displays a contour from the $\mathrm{H} \alpha$ filter image that was continuum-subtracted utilizing a technique adapted from E. L. Radice (http://www. arciereceleste.it/tutorial-pixinsight/cat-tutorial-eng/85-enhance-galaxy-ha-eng), whereby the median values of a dimmed $\mathrm{R}$ frame were subtracted from the $\mathrm{H} \alpha$ frame to remove residual red light outside of the $\mathrm{H} \alpha$ band.

Extended Data Fig. 2 shows a composite of optical images made using data from the Canada France Hawaii Telescope (CFHT), described below, and previously published ${ }^{41}$ data from the Maryland-Magellan Tunable Filter ${ }^{43}$ (MMTF) on the Magellan-Baade 6.5-metre telescope. The advantage of the CFHT is its wide field of view, which allowed us to image a square degree centred on NGC 5128 and capture both the north and south transition regions, while the MMTF offers better resolution and higher image fidelity. The CFHT data were obtained using a combination of broadband and narrowband filters with the Megacam instrument, via director's discretionary time (Principal Investigator: M.S.C.; Proposal ID: 18AD88). Observations at $\mathrm{H} \alpha$ (Ha.MP9603; wavelength range $\Delta \lambda=104 \AA$ ) were split into $5 \times 870 \mathrm{~s}$ exposures with a large dithering pattern (LDP1) and queued between 6 and 18 June 2018, resulting in a total exposure time of 4,350 s for this filter. An additional $5 \times 120 \mathrm{~s}$ exposures of archival $r$-band data (r.MP9601; $\Delta \lambda=1,480 \AA$ ) used in this paper were retrieved from the Canadian Astronomy Data Centre (Proposal ID: 03AE01).

The data reduction was performed using the Elixer pipeline $e^{44}$ to create bad-pixel masks and correct for bias and flat fielding. Photometric calibration of the new and archival CFHT data was done with MegaPipe ${ }^{45}$ using photometric standards that overlapped with Panoramic Survey Telescope and Rapid Response System coverage whenever possible, or using images taken during the same observing runs, to set up a zero-point. The $\mathrm{H} \alpha$ photometric calibration is accurate to approximately $0.05 \mathrm{mag}$, and less for the archival $r$-band data. Astrometric calibrations are based on Gaia data and have errors of $\sim 30$ mas. The images were all registered to the same pixel grid and co-added using SWarp ${ }^{46}$. Background removal was performed using a local $128 \times 128$-pixel mesh.

The large-scale CFHT image shown in Extended Data Fig. 2 is a continuum-subtracted $\mathrm{H} \alpha$ map, meant to exclusively highlight the extended line emission throughout the entire square-degree field of view. The continuum level 
in the $\mathrm{H} \alpha$ map was estimated from the broader $r$-band filter and then subtracted after matching their point spread functions (PSFs). To model the PSF in both narrowband and broadband images, we extracted $25 \times 25$-pixel image cutouts centred on source catalogues produced via SExtractor ${ }^{47}$, each normalized by the total flux in the cutout, and used the median of these normalized cutouts to create an 'average star'. The average star in both bands was re-centred and fit by a two-dimensional Moffat function to model the PSF with $(\mathrm{H} \alpha$ : amplitude $=0.034$, core width $\gamma=3.58$, power index $\alpha=2.37$; $r$-band: amplitude $=0.017$, core width $\gamma=5.70$, power index $\alpha=2.68$ ). We used the photutils (https://photutils. readthedocs.io/en/stable/psf.html) routine create_matching_kernel with a cosine bell window function to calculate the ratio of Fourier transforms needed to convolve with the $\mathrm{H} \alpha$ map to match the broader PSF of the $r$-band image. The resulting convolved $\mathrm{H} \alpha$ map was then subtracted by the $r$-band image, scaled by an arbitrary value to match the peak of the average star in both images, to produce a continuum-subtracted $\mathrm{H} \alpha$ map. The asymmetric residuals present after the continuum subtraction can be attributed to distortions over the entire field of view due to the difficulty of achieving sub-pixel astrometric alignment over a square degree and 36 individual charged coupled devices. In Extended Data Fig. 2, these negative residuals were clipped and set to zero and the image was stretched with arcsinh scaling to enhance the visualization of emission and suppress noise.

\section{Evidence for self-regulated feedback and feeding via chaotic cold accretion.} There are a range of numerical models and zoom simulations, implementing AGN feedback in various ways ${ }^{48-52}$, which can reproduce many of the observed features of the galaxy population. The widely adopted paradigm in the literature is that there are two modes of accretion (excluding hierarchical mergers) that lead to black-hole growth; the hot and the cold mode, based on whether the source of accretion fuel is hot or cold gas. Hot-mode accretion predicts inflows and outflows that are mono-phase, continuous and quiescent, whereas in the cold mode accretion occurs in more intensive and intermittent bursts, at rates that can be orders of magnitude higher than the Bondi ${ }^{53}$ rate $^{54}$. Large statistical samples ${ }^{55}$, however, have shown that the underlying dichotomy is a result of the radiation efficiency of the AGN and that both hot and cold gas have roles to play in feeding and feedback ${ }^{56}$.

Hybrid models, which have the AGN cycling between the two modes of accretion, can explain observations of black-hole masses and related galactic properties ${ }^{57}$. In these models, the steady and smooth flows described by Bondi accretion are disrupted by cooling instabilities, which result in $\mathrm{CCA}^{9,10,58,59}$ of gas onto the black hole and an ensuing self-regulated feedback loop that prevents the establishment of catastrophic cooling flows ${ }^{60}$. The unified model of AGN feeding and feedback ${ }^{17}$, which incorporates CCA as the feeding mechanism, provides a number of clear observational predictions such as multiphase gas, turbulence, inflows and outflows and so on, which have been probed by severa observations ${ }^{9,16,61}$, and provides a framework that unifies the extreme range in spatial scale over which the feedback loop exists ${ }^{10,17}$.

The observed properties of Centaurus A on micro, meso and macro scales are well described by the unified CCA model of AGN feeding and feedback. As described in the main article and below in our discussion on consistency with other observations, the galaxy is surrounded by multiphase gas clouds with a wide range of temperatures, sizes and velocity dispersions. It is possible that some of this gas (such as the ring of $\mathrm{H} \mathrm{I}$ at $\sim 10 \mathrm{kpc}$ scales) is left over from previous tidal interactions with infalling galaxies, but CCA provides a robust mechanism for the replenishment of such clouds, which we show are destroyed on relatively short timescales by interactions with AGN outflows. There are also warm optical filaments and evidence for inflows and outflows on small, intermediate and large scales (Table 1 and discussion below). Very-long-baseline interferometry (VLBI) observations indicate considerable variability on the micro scales of the radio jet and the sharp boundaries of the inner lobes suggest that they are being inflated by a fresh outburst of activity not directly connected to the powering of the NML, implying that AGN activity is intermittent, with a duty cycle that is able to sustain a broad transonic outflow that pervades the meso and macro scales, causing weather systems in the NML and turbulent filaments in the outer lobes (the outflow may also be augmented by supernovae-driven winds as discussed below in relation to simulations). These observational data, describing an intermittent AGN with multiphase inflows and outflows, clearly rule out a purely hot-mode accretion model and justify the application of the unified CCA model of AGN feeding and feedback to Centaurus A throughout this paper.

AGN feedback outflow/inflow calculations. Our calculations are based on the equations of Gaspari et al. ${ }^{17}$, hereafter G17, who have used the CCA model of AGN feedback ${ }^{10}$ to unify the ten-order-of-magnitude range in physical scale for energy transfer in active galaxies ${ }^{9}$ by leveraging a wide range of high-resolution simulations and multi-wavelength observations. We interpret the long radio arc to the south of the Centaurus A core, shown in Fig. 1, as the thermalization radius, $r_{\text {th }}$ where the outflow pressure equalizes with the interstellar-medium pressure. We measure $r_{\text {th }}$ as approximately $75 \mathrm{kpc}$ and estimate that the outflow has spread to a width of $90 \mathrm{kpc}$ at a radius of $63 \mathrm{kpc}$, giving an opening angle of $71^{\circ}$ (as shown in Extended Data Fig. 1, right panel), resulting in a solid angle for the bipolar conical outflow of $\Omega \approx 2.3 \mathrm{sr}$. In this scenario, we are viewing the edge of a segment of a sphere that has its centre at the position of the AGN. As such, the estimate of $r_{\text {th }}$ will not have a large dependence on the inclination of the outer lobes (or the inner jets and lobes). In any case, our derived quantities are not overly sensitive to the exact value of $r_{\text {th }}$ and uncertainties of up to $10 \%$ do not affect our conclusions.

We use equation (26) in G17 for the thermalization radius (in kpc), including the dependence on the bipolar outflow solid angle $\Omega$ in sr:

$$
r_{\text {th }} \simeq 55 \Omega_{4 \pi}^{-1} T_{\mathrm{x}, 7.4} \simeq 295 T_{\mathrm{x}, 7.4}
$$

where $T_{\mathrm{x}, 7.4} \equiv T_{\mathrm{x}} / 10^{7.4} \mathrm{~K}$ is the $\mathrm{X}$-ray temperature of the macro-scale hot halo $\left(T_{\mathrm{x}}\right)$, normalized to units of $10^{7.4} \mathrm{~K} \simeq 2.16 \mathrm{keV}$ and $\Omega_{4 \pi} \equiv \Omega / 4 \pi$.

Rearranging equation (1) to compute a value for the macro $T_{\mathrm{x}}$ using our constrained $r_{\text {th }} \simeq 75 \mathrm{kpc}$, we obtain $T_{\mathrm{x}} \simeq 0.55 \mathrm{keV}$, which is similar to other X-ray constraints (see below). Following equation (18) in G17, the micro-scale outflow velocity in $\mathrm{km}$ per s, $v_{\text {out }} \simeq\left(1.4 \times 10^{4}\right) T_{\mathrm{x}, 7.4}^{1 / 2}$, can be determined directly from the macro X-ray temperature, giving us $v_{\text {out }} \simeq 7,060 \mathrm{~km} \mathrm{~s}^{-1}$.

We proceed by calculating the hot plasma entrainment factor, $\eta_{\text {hot }}$, by also expanding equation (23) in G17 to include the solid angle term $\Omega_{4 \pi}$ and central (within radius $r_{0} \approx 1 \mathrm{kpc}$ ) density normalized to units of $10^{-25} \mathrm{~g} \mathrm{~cm}^{-3}$, $\rho_{0,25} \equiv \rho_{0} / 10^{-25} \mathrm{~g} \mathrm{~cm}^{-3}$ (where $\rho_{0}$ is density in $\mathrm{g} \mathrm{cm}^{-3}$ ), such that:

$$
\eta_{\mathrm{hot}}=40 T_{\mathrm{x}, 7.4}^{-1}\left(\Omega_{4 \pi} \rho_{0,25} r_{\mathrm{kpc}}\right)^{2 / 3}=9.4 T_{\mathrm{x}, 7.4}^{-1} r_{\mathrm{kpc}}^{2 / 3}
$$

where $r_{\mathrm{kpc}}$ is the radial distance from the nucleus (in kpc); in the last step, we have used the observed ${ }^{62}$ central hot-plasma density of NGC $5128, \rho_{0 \text { hot }} \simeq 6 \times 10^{-26} \mathrm{~g} \mathrm{~cm}^{-3}$ (which is also close to the average assumed in G17) at a radius $r_{\text {kpc }}=0.5 \mathrm{kpc}$. By using the retrieved macro $T_{\mathrm{x}} \simeq 0.55 \mathrm{keV}$ in equation (2), we obtain an entrainment factor $\eta_{\text {hot }} \simeq 42$. This is the loading factor by which mass is entrained by the micro outflow to produce the observed macro outflow. The macro-outflow velocity $v_{\text {OUT }}$ is related to the micro-outflow velocity and the entrainment factor by equation (21) in G17, $v_{\mathrm{OUT}}=v_{\text {out }} / \eta_{\text {hot' }}^{1 / 2}$, providing $v_{\text {OUT }} \simeq 1,090 \mathrm{~km} \mathrm{~s}^{-1}$.

Further, we use these values to compute the macro/micro mass-outflow and mass-inflow rates. The macro mass-inflow rate, also known as the (quenched) cooling rate, $\dot{M}_{\text {cool }}$, in units of solar masses per yearis also tied to the macro X-ray temperature via equation (7) in G17, $\dot{M}_{\text {cool }} \simeq 1.1 T_{\mathrm{x} 74}^{2}$, which gives us $\dot{M}_{\text {cool }}=0.07 \mathrm{M}_{\odot} \mathrm{yr}^{-1}$. As discussed in G17, only a small percentage $(\sim 3 \%)$ of the inflowing mass is actually accreted through the black-hole horizon. Most of the inflowing mass is expelled via the generated micro-scale outflow (converting binding energy into mechanical feedback energy), such that the micro mass-outflow rate is $\dot{M}_{\text {out }} \approx \dot{M}_{\text {cool }}$. The macro mass-outflow rate, $\dot{M}_{\text {OUT }}$, is then given by the micro mass-outflow rate multiplied by the entrainment factor (equation (19) in G17); for our NGC 5128 outflow, $\dot{M}_{\text {OUT }}=\eta_{\text {hot }} \dot{M}_{\text {out }} \simeq 2.9 M_{\odot} \mathrm{yr}^{-1}$. To complete the loop and finally link the macro and micro scales, we calculate the micro mass-inflow rate (that is, the accretion rate onto the black-hole horizon) by equation (12) in G17 as $\dot{M}_{\bullet}=\left(3 \% \dot{M}_{\text {cool }}\right) T_{\mathrm{x}, 7.4} \simeq 5.3 \times 10^{-4} M_{\odot} \mathrm{yr}^{-1}$. These values are tabulated in Table 1. See below for a discussion on the consistency of these calculations with observations at all wavelengths and physical scales.

Consistency with other observations and theory. In this work, we have used low-frequency radio observations to identify evidence for a broad, large-scale outflow from the AGN of Centaurus A. Similar radio features to the southern radio arc, resulting from explosive outbursts at the centre of our own Galaxy, have been observed on smaller scales $(\sim 400 \mathrm{pc})$ at $\mathrm{GHz}$ frequencies ${ }^{63}$. On more comparative scales (several kpc), the situation may be more analogous to the Fermi bubbles ${ }^{64,65}$ observed in $\gamma$-rays above and below the Galactic plane, with corresponding polarized $\mathrm{GHz}\left(\right.$ ref. ${ }^{66}$ ) and microwave ${ }^{67}$ radio emission. These bubbles can be explained by a weak but sustained outflow from the Galactic Centre ${ }^{68}$, where the expelled gas is unable to escape the Galaxy. In contrast, the AGN-driven Centaurus A outflow is much more powerful, resulting in AGN feedback effects that extend ten orders of magnitude in physical scale, well beyond the optical extent of the galaxy. The recent discovery by the eRosita X-ray telescope ${ }^{69}$ of bubbles that are even larger and more energetic than the Fermi bubbles in the Milky Way ${ }^{70}$ indicates that our own Galaxy may have once harboured an AGN, however the range of its influence would still have been markedly less than in the case of Centaurus A.

We have used the G17 equations for the unified CCA model of AGN feedback to calculate the properties of the large-scale outflow, based on our radio image. From these large-scale (macro) properties, we have inferred the small-scale (micro) characteristics of the AGN outflow and inflow. Here we discuss the observational and energetic implications of these calculations and check for consistency with observations from the macro scale, through the intermediate (meso) scale and down to the micro scale (Table 1).

Macro. As discussed above, we derive a hot-halo X-ray gas temperature of $T_{\mathrm{x}} \simeq 0.55 \mathrm{keV}$ for our measured thermalization radius of $r_{\mathrm{th}} \simeq 75 \mathrm{kpc}$. Current published X-ray observations of NGC 5128 lack the field of view required to image out to the thermalization radius. However, using the much smaller fields of view of ROSAT (out to $\sim 35 \mathrm{kpc}$ ) and Chandra (out to $\sim 1 \mathrm{kpc}$ ), the hot-halo temperature has been estimated at $0.3 \mathrm{keV}\left(\right.$ ref. ${ }^{20}$ ) and $0.6 \mathrm{keV}\left(\right.$ ref. $\left.{ }^{71}\right)$, respectively. Our value is 
within the bounds of these two estimates made with limited fields of view. Within the southern $\sim 1 \% \sim 60 \mathrm{kpc}$ radius, which is free from clouds of cold gas, no features in the radio band or at other wavelengths (apart from the diffuse X-ray halo) are necessarily expected ${ }^{16,72,73}$. With a larger field of view, forthcoming eRosita ${ }^{69}$ data may show X-ray features due to shocks at the thermalization radius where we observe the southern radio arc.

Using our derived outflow properties, we can derive some constraints on the energy budget of the system. We note here that the G17 model describes the final quasi-isotropic macro feedback deposition, mainly via wide entrained outflows and large cavities. In this model, narrow jet features can coexist or even power the large-scale outflows, but their micro effects are not considered. Our analysis therefore does not include the micro-scale effects of the Centaurus A jets, but we do take into account that, on intermediate scales, previous jet outbursts have carved out a cocoon in the surrounding interstellar medium/intergalactic medium, into which the outflow we detect is funneled. We first calculate the macro-outflow power as:

$$
P_{\text {OUT }}=\frac{1}{2} \dot{M}_{\text {OUT }} v_{\text {OUT }}^{2} \simeq 1.1 \times 10^{42} \mathrm{erg} \mathrm{s}^{-1} .
$$

The pressure at the thermalization radius, $p_{\mathrm{th}}$, is then given by:

$$
p_{\mathrm{th}}=\frac{P_{\mathrm{OUT}}}{v_{\mathrm{OUT}} A_{\mathrm{th}}} \simeq 1.4 \times 10^{-13}{\mathrm{dyn} \mathrm{cm}^{-2}}
$$

where $A_{\mathrm{th}}$ is the surface area of the spherical cap at radius $r_{\text {th }}$, defined by the cone with solid angle $\Omega=1.15 \mathrm{sr}$, as measured for the outflow to the south of the galaxy. We then calculate the energy of this bubble, $E_{\text {bubble }}$, as:

$$
E_{\text {bubble }}=4 p_{\text {th }} V_{\text {th }} \simeq 2.7 \times 10^{57} \mathrm{erg},
$$

where $V_{\text {th }}$ is the volume of the spherical sector defined by $\Omega=1.15 \mathrm{sr}$. This is the macro feedback energy being transmitted to the interstellar medium/intergalactic medium (strictly speaking, a lower limit, since it ignores shocks ${ }^{74}$ ). For our outflow with power $P_{\text {OUT }} \simeq 1.1 \times 10^{42} \mathrm{erg} \mathrm{s}^{-1}$, the broad outflow would take $\sim 1.6 \mathrm{Gyr}$ to deposit this amount of energy. Previous dynamical age estimates of the outer lobes, on the order of $\sim 1 \mathrm{Gyr}$ (refs. ${ }^{75,76}$ ), have been very sensitive to the inclination angle of the lobes to our line of sight. It is estimated from VLBI observations that the AGN jets are inclined at an angle $50<\theta_{\mathrm{LOS}}<80^{\circ}$ (ref. ${ }^{13}$ ), where $\theta_{\mathrm{LOS}}$ is inclination angle. If we assume that the southern radio arc represents the edge-on view of the compression zone, then our estimate of the thermalization radius (and therefore the age of the outflow) is independent of the inclination angle of the jets. So, adopting the flow-driven model for the outer lobes ${ }^{75}$, where the age is given by $t_{\text {lobes }}=600 / \cos \theta_{\text {LOS }}$ Myr, and using our age estimate for the macro outflow as the outer lobe age, then we arrive at an estimate for the inclination angle of $\theta_{\mathrm{LOS}}=68^{\circ}$, in agreement with VLBI observations.

The thermal gas present in the outer lobes has been measured by studies of radio polarization ${ }^{77}$ and $\mathrm{X}$-rays ${ }^{78}$. The number density of thermal gas derived from these observations is $\sim 10^{-4} \mathrm{~cm}^{-3}$. If this thermal gas is due to entrainment of gas from the central galaxy, then the implied mass-outflow rate is $\sim 20 M_{\odot} \mathrm{yr}^{-1}$, an order of magnitude greater than our inferred mass-outflow rate (Table 1). It is possible, however, that these observational results are contaminated by Galactic foreground emission, a scenario that could not be ruled out by the authors ${ }^{77,78}$, and has also been put forward as an explanation by other authors who estimate a much smaller thermal gas density of $\sim 10^{-8} \mathrm{~cm}^{-3}$ for the outer lobes, based on entrainment by the currently active inner jets ${ }^{76}$. Our mass-outflow rate of $\sim 2.9 M_{\odot} \mathrm{yr}^{-1}$ based on a broad AGN outflow is therefore consistent with the scenario where the true thermal gas content of the outer lobes is on the order $\sim 10^{-5} \mathrm{~cm}^{-3}$ and the remainder observed by radio polarization and X-ray studies ${ }^{77,78}$ is due to Galactic foreground contamination

Meso. We now turn our attention to the intermediate (meso) scales and in particular to the north of the galaxy, where the situation is vastly different from the south. This contrast is due to the presence of clouds of cold and warm gas in the path of the AGN outflow, as we have shown in Fig. 2. The purple contour in Fig. 2 shows $\mathrm{H}$ I emission ${ }^{22}$, which is prominent in the disk of NGC 5128 but also exists in a ring-like structure surrounding the galaxy at a radius $\lesssim 17 \mathrm{kpc}$ (ref. ${ }^{79}$ ). The $\mathrm{H}$ i ring appears to be tilted on a similar angle to the radio jets and is moving such that the cloud to the north (near the centre of Fig. 2) is coming toward us with a line-of-sight velocity of around $400 \mathrm{~km} \mathrm{~s}^{-1}$ and the cloud to the south of the galaxy is moving away from us at around $700 \mathrm{~km} \mathrm{~s}^{-1}$ (ref. ${ }^{79}$ ). At the centre of Fig. 2, the eastern edge of the northern $\mathrm{H}$ i cloud has entered the outflow from the AGN, which has been funneled into the cavity created by previous outbursts of radio-jet activity $^{21}$. As the cold gas impacts the outflow, an area of turbulence results (evident in the large random velocities observed in the filaments $\mathrm{s}^{26,80-82}$ ), inducing star formation ${ }^{83}$ and creating the distinctive ionized filament, which is bright in $\mathrm{H} \alpha$

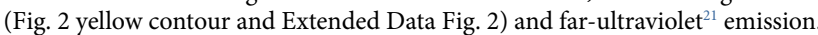

While the differences in the environments to the north and south of the galaxy can be tied to its merger history, asymmetries in the warm and cold gas distributions around galaxies can occur in the absence of a merger event, as has been shown in simulations ${ }^{84}$, resulting in one part of a bipolar outflow being hampered or deflected. At smaller radii in NGC $5128(\sim 3.5 \mathrm{kpc})$ there is evidence to suggest a sharp discontinuity in pressure due to non-hydrostatic gas motions or 'sloshing' to the north of the galaxy ${ }^{85}$, helping to explain asymmetries in the gas distribution and radio morphology of Centaurus A.

The outflow parameters we derive are very similar to those of the 'starburst wind' invoked by Neff et al. ${ }^{21}$ to explain the features of the NML. Above we calculated an outflow power of $P_{\text {OUT }} \simeq 1 \times 10^{42} \mathrm{erg} \mathrm{s}^{-1}$, the same value used by Neff et al. ${ }^{21}$ to estimate the power incident on the small gas cloudlets making up the inner and outer filaments. They find that this wind alone is insufficient to provide all the necessary power ${ }^{81,83}$ to sustain the emission-line filaments, but could provide enough power if boosted by a factor of 10 by the AGN jet flow. The current AGN jet power is estimated as $P_{\text {jet }} \simeq 2 \times 10^{43} \mathrm{erg} \mathrm{s}^{-1}$ (ref. ${ }^{8}$ ), so it is not unreasonable to assume that similar powers have been available in previous AGN outbursts to boost the wind in such a manner, close to the jet axis. While the G17 equations, which focus on the macro feedback properties, do not describe such localized and narrow jet features, this scenario is still consistent with the CCA model and the broad outflow that we have identified. Given the close alignment of the emission-line filaments with the orientation of the inner lobes and jets, it is possible that the outflow could be sporadically boosted by a factor of 10 in power by a jet outburst, providing power to these short-lived (10-15 Myr; ref. ${ }^{21}$ ) clouds, without the need for a 'large-scale jet' currently connecting the inner lobes to the NML. However, more detailed simulations incorporating both broad winds and narrow jets impacting cold gas clouds are required to verify these claims.

The centre of Fig. 2 also appears to be the nexus of the X-ray ${ }^{20}$ (red contours) and radio (this work; blue contours and greyscale) knots. The $\mathrm{X}$-ray knots are analysed in detail by Kraft et al. ${ }^{20}$, hereafter K09, who conclude that the most likely source of energy for the knots is the previously discussed large-scale jet ${ }^{19}$. Since we have dispelled the existence of this large-scale relativistic jet, we assess whether our transonic large-scale outflow can power the X-ray knots. As a minimum test of consistency, K09 assert that the ram pressure of their large-scale jet must be greater than the pressure of the individual knots to contain them. Using the same values as K09 for the cloud cross-sectional area $\left(A_{\text {rodd }}\right)$, the ram pressure for our outflow is $p_{\text {ram }}=2 P_{\text {OUT }} /\left(v_{\text {OUT }} A_{\text {clouds }}\right) \simeq 5.9 \times 10^{-10} \mathrm{dyn} \mathrm{cm}^{-2}$. This is $\sim 30$ times the typical pressure of the K09 X-ray clouds, enough to prevent them from dissipating too quickly. The total thermal energy of the X-ray knots estimated by K09 is $2.6 \times 10^{55} \mathrm{erg}$. Given the outflow parameters that we calculate above, we estimate that the power intercepting the clouds is $1.4 \times 10^{40} \mathrm{erg} \mathrm{s}^{-1}$, so it would take $\sim 56 \mathrm{Myr}$ to provide the required energy to the clouds. This is an order of magnitude longer than the lifetimes of the clouds estimated by K09, of a few million years. As discussed above for the smaller emission-line clouds of the filaments and below for the micro-scale duty cycle, the AGN jets can provide sporadic boosts to the outflow that can make up the energy deficit, while the constant, broad outflow maintains the pressure required to contain the clouds.

Closer to the nucleus, but still in the meso-scale regime, other outflows of gas have been identified in Centaurus A. Relatively dense and warm outflows $\sim 3 \mathrm{kpc}$ to the east and west of the galaxy have been identified by a spectral analysis of Chandra X-ray data ${ }^{86}$, with an estimated outflow velocity of $\sim 1,000 \mathrm{~km} \mathrm{~s}^{-1}$ and mass-outflow rate of $\sim 1 M_{\odot} \mathrm{yr}^{-1}$. A slower, but potentially more massive outflow of cold neutral and ionized gas has also been detected within the inner $500 \mathrm{pc}$ of Centaurus $\mathrm{A}^{87}$, with a projected velocity of $60 \mathrm{~km} \mathrm{~s}^{-1}$ and mass-outflow rate $\sim 2 M_{\odot} \mathrm{yr}^{-1}$ (uncertain by a factor of 3 ). The outflows probably result from eruptive events near the galactic nucleus that exist on smaller timescales (for example, $\sim 3$ Myr for the hot east-west outflows ${ }^{86}$ ) and don't necessarily have the same physical origin as our large-scale wind, but demonstrate that outflows with comparative mass-outflow rates and velocities do exist in Centaurus A on these physical scales. It is interesting to note that there is currently little evidence in Centaurus A for cold outflows on scales larger than $1 \mathrm{kpc}$ (refs. ${ }^{2,88}$ ). However, the currently available $\mathrm{H}$ I observations ${ }^{22,79}$, for example, do not cover out to the thermalization radius that has been identified in this work. New instruments such as the Australian Square Kilometre Array Pathfinder ${ }^{89,90}$ and MeerKAT ${ }^{11,92}$ will soon probe these regions and may uncover so-far undiscovered cold gas clouds.

Micro. One interpretation of the sub-parsec scale VLBI data on the Centaurus A core ${ }^{13,93,94}$ is that discrete jet components with subluminal motions of $\sim 0.1 \mathrm{c}$ $\left(3 \times 10^{4} \mathrm{~km} \mathrm{~s}^{-1}\right)$ coexistent with a faster underlying jet flow with velocity $\sim 0.45 \mathrm{c}$ $\left(13.5 \times 10^{4} \mathrm{~km} \mathrm{~s}^{-1}\right)$, where $c$ is the velocity of light. X-ray observations have also been used to look for ultrafast outflows from the core of Centaurus $\mathrm{A}^{95}$. Blueshifted $\mathrm{Fe}-\mathrm{K}$ absorption lines indicate an outflow from Centaurus $\mathrm{A}$, variable with time, of between 900 and $1,500 \mathrm{~km} \mathrm{~s}^{-1}$. These velocities are less than the $\sim 10^{4} \mathrm{~km} \mathrm{~s}^{-1}$ typical velocities of ultrafast outflows found in radio-loud $\mathrm{AGN}^{95}$, but this could be due to a different angular regime being probed because of the extreme proximity of Centaurus A. In any case, our inferred micro-scale outflow velocity of $\sim 7,060 \mathrm{~km} \mathrm{~s}^{-1}$ sits comfortably between these two observed micro-outflow velocities. While we are not concerned in this work with the specific mechanisms that produce these outflows, we note here that AGN jets can produce such outflows ${ }^{96}$, resulting in feedback at the macro scale that occurs over a much broader cross section than the typical jet width ${ }^{97,98}$. 
As for the inferred inflow parameters, our results give a value of $\dot{M}_{\text {in }} \approx 5.3 \times 10^{-4} M_{\odot} \mathrm{yr}^{-1}$ for the black hole inflow rate, which is an average over the lifetime of the source and is related to the gas accreting through the black hole horizon (hence the small value). The measured inflow rate of $\mathrm{H} \mathrm{I}$ (refs. ${ }^{99,100}$ ) at the micro scale is $5 \times 10^{-2} M_{\odot} \mathrm{yr}^{-1}$ and since only $\sim 3 \%$ of the actual inflowing gas is accreted through the black-hole horizon ${ }^{17}$ these numbers are fully consistent. Interestingly, if we apply a dynamical jet model $^{48}$ to our inferred accretion rate and estimate the jet power as $P_{\text {jetmodel }}=\epsilon \dot{M}_{\bullet} c^{2}$, using a reasonable value for the jet production efficiency, $\epsilon$, of $10 \%$ (ref. ${ }^{48}$ ) we obtain $P_{\text {jet }} \simeq 3 \times 10^{42} \mathrm{erg} \mathrm{s}^{-1}$. This falls between our inferred outflow power of $P_{\text {OUT }} \simeq 1 \times 10^{42} \mathrm{erg} \mathrm{s}^{-1}$, which is a measure of the average power over the lifetime of the outflow, and the estimated current jet power $P_{\text {jet }} \simeq 2 \times 10^{43} \mathrm{erg} \mathrm{s}^{-1}$ (ref. ${ }^{8}$ ). This indicates that our inferred values are reasonable and in agreement with a model ${ }^{48}$ that invokes an AGN that intermittently switches on and off. A rough estimate for the duty cycle of the AGN activity can be obtained by calculating the fraction of 'on' time needed to keep the hot halo in approximate heating-cooling balance. We estimate this by taking the ratio of the X-ray cooling luminosity (calculated from equation (7) in G17 using our inferred value for $T_{\mathrm{x}} \simeq 0.55 \mathrm{keV}$, we obtain $L_{\mathrm{x}} \simeq 1 \times 10^{41} \mathrm{erg} \mathrm{s}^{-1}$ ) and the jet power, $P_{\text {jet }} \simeq 2 \times 10^{43} \mathrm{erg} \mathrm{s}^{-1}$, which gives us a value of $5 \%$. This is a reasonable value, which we note is well above the duty cycle that would be inferred from the mass-luminosity scaling relationship ${ }^{101}$ of $0.1 \%$ (based on a black-hole mass of $0.35-8.5 \times 10^{7}$ (ref. ${ }^{102}$ ) and a $1.4 \mathrm{GHz}$ radio luminosity of $2.3 \times 10^{24} \mathrm{~W} \mathrm{~Hz}^{-1}$ $\left(\right.$ ref $\left.{ }^{103}\right)$ ), because this scaling relationship averages across all black holes of the same mass, most of which will have radio-quiet host galaxies, unlike Centaurus A.

We can also calculate the (micro) mechanical efficiency as

$\epsilon_{\mathrm{BH}}=P_{\mathrm{OUT}} /\left(\dot{M}_{\mathrm{in}} c^{2}\right)=0.036$. We note that this value is consistent with the $3 \pm 1 \%$ found via general-relativistic, radiative magneto-hydrodynamic simulations over a wide range of tested physics ${ }^{72}$ and is far from the unity value needed to power relativistic jets via spin (for example, refs. ${ }^{104,105}$ ). Indeed, such a large value would overheat and unbind most of the group halos.

Implications for cosmological simulations. Feedback (through supernovae-driven winds and black-hole accretion) affects galaxy evolution and cosmology, but is difficult both to simulate and to observe $e^{3,4,106}$, which is due in no small part to the range of physical scales involved. Accretion onto black holes occurs on scales of $\sim 10^{-5} \mathrm{pc}$ while the energy-driven outflows that this process launches imprint their signatures on scales of $\sim 10^{5} \mathrm{pc}$, affecting the density, temperature, entropy and cooling times into the far reaches of the gas halo ${ }^{107}$. In fact, the effects of supermassive black-hole growth and associated feedback extend beyond the halo scale through cosmic gas flows and supermassive black hole-supermassive black hole mergers ${ }^{108,109}$, such that any simulation conducted in cosmic isolation will be incomplete. Hence, we turn our attention here to cosmological simulations and focus on how observations of Centaurus A (presented here and planned for the future) can be used to inform simulations (and vice versa).

Broad, bipolar galactic-scale outflows are a feature of modern cosmological simulations such as EAGLE ${ }^{110}$, SIMBA $^{111}$ and Horizon-AGN ${ }^{112}$. In these simulations, both AGN and stellar feedback contribute to the outflows and, in the case of AGN-driven feedback, the directionality of the bipolar outflows along the minor axis of the galaxies are directly specified as part of the sub-grid physics implemented. Higher-resolution zoom simulations such as Romulus $\mathrm{C}^{113}$ have been able to reproduce collimated large-scale outflows from isotropic AGN feedback introduced on small scales, showing that this emergent effect is a result of the morphology and angular momentum of the gas at the base of the outflow and not due to jet orientation. Such AGN feedback effects are difficult to implement in a full-scale cosmological simulation, as limited resolution affects the way that AGN outflows couple to nearby gas particles ${ }^{113}$.

A current set of key cosmological simulations that include AGN feedback is the IllustrisTNG suite $\mathrm{e}^{114-118}$. The simulation runs TNG100 and TNG300 produced realizations of very large volumes $\left(\sim 100^{3}\right.$ and $\sim 300^{3}$ comoving $\mathrm{Mpc}^{3}$, respectively), allowing for statistical studies of large galaxy populations, at the cost of resolution to study smaller details of individual galaxies. The simulation run TNG50 (refs. ${ }^{3,119}$ ) simulated a smaller volume at a higher resolution, making it ideal to study feeding and feedback processes. The resolution of TNG50 is equivalent to or better than many 'zoom' simulations that have previously identified fast galactic outflows of $\sim 1,000 \mathrm{~km} \mathrm{~s}^{-1}$ out to radii of $100 \mathrm{~s}$ of $\mathrm{kpc}$ (ref. ${ }^{120}$ ), but also allows the study of feedback effects on cosmological scales.

An important result from TNG50 is that large-scale outflows are bipolar, with wide opening angles (for example, around $70^{\circ}$ ), directed along the minor axis of the galaxy ${ }^{3}$. This is a purely emergent property of the outflows, since the feedback inputs inserted at small scales (associated with both supernovae-driven winds and black-hole accretion) have no direction dependence. Our Centaurus A observations are consistent with this scenario, that is, despite the radio galaxy being characterized by highly collimated, narrow jets on sub-parsec scales, the end result is a broad bipolar outflow affecting a wide region along the minor axis of the galaxy. This also corroborates the assumption of the CCA model that broad, large-scale outflows dominate over the effects of narrow jets at scales of $10 \mathrm{~s}$ to $100 \mathrm{~s}$ of kiloparsecs.

Another important result from TNG50 is that outflows are multiphase, consisting of cold, warm and hot gas. Observational examples where multiple phases are observed in the one source, however, are rare ${ }^{3}$, hence the importance of Centaurus A, which provides a rich dataset of multiphase gas observations. Of critical importance to the CCA model and cosmological simulations are the X-ray scaling relations, which allow many properties to be inferred from the hot-gas halos of galaxies ${ }^{54}$. As discussed previously, X-ray observations of the hot-gas halo of Centaurus A have been limited by instrumental fields of view. Our radio observations, however, have allowed us to infer the hot-halo temperature via a measurement of the thermalization radius. While outside of the scope of this work, subsequent studies will therefore be able to compare the observed large and small scale, multiphase gas properties of Centaurus A with analogues of the galaxy found in simulations such as TNG50.

Compatibility with observations at higher redshift. The general consistency of the outflow properties of Centaurus A with galaxies throughout the IllustrisTNG simulations gives us some confidence that Centaurus A is not atypical in terms of its feedback processes. This is also supported by comparisons with galactic outflows observed in galaxies at higher redshifts. It is clear that outflows, particularly driven by stellar winds and supernovae, are ubiquitous in galaxies throughout the Universe and across cosmic time ${ }^{2}$. These stellar processes must contribute to the observed outflow in Centaurus A, which is currently undergoing a starburst ${ }^{21}$, but energy from the AGN is required to reproduce the observed features in the transition regions. While it is not possible to separate the contribution of AGN activity and stellar activity in either simulations or observations ${ }^{3}$, there is now a lot of evidence for outflows in AGN, obtained mainly through optical absorption and emission lines and X-ray observations.

In the local Universe ( $z \simeq 0$ to $z \simeq 0.2$, where $z$ is redshift), ionized outflows with velocities ranging from $1,000 \mathrm{~km} \mathrm{~s}^{-1}$ to $0.4 \mathrm{c}$ are observed in up to $50 \%$ of radio-loud $\mathrm{AGN}^{95,121,122}$. These identifications via measurement of $\mathrm{Fe}-\mathrm{K}$ absorption lines in the X-ray band favour the discovery of very fast outflows close to the nucleus, so slower outflows further out may be missed by this method. These ultrafast outflows on the order $10^{4} \mathrm{~km} \mathrm{~s}^{-1}$ are consistent with our inferred micro-outflow rate for Centaurus A of $7,060 \mathrm{~km} \mathrm{~s}^{-1}$. These observations demonstrate that the presence of AGN jets does not preclude the existence of broad outflows or winds, as we observe in Centaurus A. This has also been shown by the identification of an $\simeq 1,000 \mathrm{~km} \mathrm{~s}^{-1}$ outflow in the powerful local radio galaxy 3C 120 (ref. ${ }^{123}$ ).

At higher redshifts $(z>0.6)$, optical emission lines provide a means to identify outflows. Velocity widths of $>600 \mathrm{~km} \mathrm{~s}^{-1}$ indicate fast ionized outflows in $50-70 \%$ of AGN and are more prevalent in radio-loud AGN ${ }^{124}$. Around the peak of quasar space density at $z \simeq 2$ (ref. ${ }^{125}$ ), outflows are also found to be common in galaxies harbouring $\mathrm{AGN}^{126-128}$. These outflows, also detected by means of optical emission lines, are found to have velocities from 300 to $3,000 \mathrm{~km} \mathrm{~s}^{-1}$. Gravitational lensing has allowed glimpses of ionized outflows with similar properties out to $z=3.91$ (refs. ${ }^{121,122}$ ), and even out to $z \simeq 5$ there is evidence for molecular outflows in quasars, which appear to be dominated by AGN activity rather than stellar processes $^{129}$.

It is clear that outflows such as those observed in Centaurus A are a common and important feature of galaxies across cosmic time. However, their detection and characterization is difficult, usually relying on a single tracer and incorporating many assumptions about geometry and galactic properties ${ }^{2,3}$. While we should be careful comparing Centaurus A directly with high redshift galaxies, it does bear important similarities to radio galaxies at high redshifts (for example, residing in a group and being recently post-merger, unlike most galaxies in the local Universe). The availability of a multitude of different tracers and the ability to observe both micro- and macro-physics in the one source provide our best opportunity to study physical processes that are extremely difficult to observe for more distant sources, such as outflow launching mechanisms and multiphase gas interactions, and to incorporate these processes into simulations in a coherent and self-consistent manner.

Future developments. We are still at the beginning of our journey to understand the details of AGN feeding and feedback and implement them into cosmological simulations in a complete sense. Centaurus A presents a unique opportunity for us to use a multi-wavelength, multiscale approach, combining theory and observations, to thoroughly understand the multiphase inflows and outflows that are ubiquitous across the Universe. As observations move toward higher resolution and sensitivity and the challenges of wide-field imaging are addressed, our data on Centaurus A will continue to improve, allowing us to fill in the many gaps in our Table 1. Detailed and accurate simulations spanning at least ten orders of magnitude (and incorporation of models into cosmological simulations), are required if we are to fully make sense of these data. How is energy transported across the various scales and what are the duty cycles for feedback, and how does this correlate to galaxy evolution over cosmic time? These questions and many more ${ }^{9}$ can be tackled using the unique resource that is our nearest radio galaxy.

\section{Data availability}

The raw visibility data from the MWA that support the findings of this study (as detailed in Table 1) are publicly available from the MWA All-Sky Virtual Observatory; https://asvo.mwatelescope.org/. The MWA radio image data (as displayed in Figs. 1 and 2 and Extended Data Fig. 1) can be accessed through 
the Strasbourg Astronomical Data Center (CDS) via anonymous ftp to cdsarc.u-strasbg.fr (130.79.128.5) or via https://cdsarc.unistra.fr/viz-bin/cat/J/ other/NatAs. Optical image data require specialist instrumental knowledge for accurate interpretation and will be made available upon reasonable request to the corresponding author, so that additional guidance can be provided.

\section{Code availability}

No custom code or algorithm was developed as part of this work, apart from simple scripting routines written in the Python language and used to run standard astronomy software tools, as described in the text.

Received: 29 April 2021; Accepted: 1 November 2021; Published online: 22 December 2021

\section{References}

1. King, A. \& Pounds, K. Powerful outflows and feedback from active galactic nuclei. Annu. Rev. Astron. Astrophys. 53, 115-154 (2015).

2. Veilleux, S., Maiolino, R., Bolatto, A. \& Aalto, S. Cool outflows in galaxies and their implications. Astron. Astrophys. Rev. 28, 2 (2020).

3. Nelson, D. et al. First results from the TNG50 simulation: galactic outflows driven by supernovae and black hole feedback. Mon. Not. R. Astron. Soc. 490, 3234-3261 (2019)

4. Heckman, T. et al. COS-burst: observations of the impact of starburst-driven winds on the properties of the circum-galactic medium. Astrophys. J. 846, 151 (2017).

5. Israel, F. P. \& Centaurus, A.- Centaurus A-NGC 5128. Astron. Astrophys. Rev. 8, 237-278 (1998).

6. Feain, I. J. (ed.) The many faces of Centaurus A. Publ. Astron. Soc. Aust. 27(Special issue), 379-495 (2010)

7. Feain, I. J. et al. The radio continuum structure of Centaurus A at $1.4 \mathrm{GHz}$. Astrophys. J. 740, 17 (2011).

8. Neff, S. G., Eilek, J. A. \& Owen, F. N. The complex north transition region of Centaurus A: radio structure. Astrophys. J. 802, 87 (2015).

9. Gaspari, M., Tombesi, F. \& Cappi, M. Linking macro, meso, and micro scales in multiphase AGN feeding and feedback. Nat. Astron. 4, 10-13 (2020).

10. Gaspari, M., Ruszkowski, M. \& Oh, S. P. Chaotic cold accretion on to black holes. Mon. Not. R. Astron. Soc. 432, 3401-3422 (2013).

11. Harris, G. L. H., Rejkuba, M. \& Harris, W. E. The distance to NGC 5128 (Centaurus A). Publ. Astron. Soc. Aust. 27, 457-462 (2010).

12. Clarke, D. A., Burns, J. O. \& Norman, M. L. VLA observations of the inner lobes of Centaurus A. Astrophys. J. 395, 444 (1992).

13. Tingay, S. J. et al. The subparsec-scale structure and evolution of Centaurus A: the nearest active radio galaxy. Astron. J. 115, 960-974 (1998).

14. Janssen, M. et al. Event Horizon Telescope observations of the jet launching and collimation in Centaurus A. Nat. Astron. 5, 1017-1028 (2021).

15. Tingay, S. J. et al. The Murchison Widefield Array: the Square Kilometre Array precursor at low radio frequencies. Publ. Astron. Soc. Aust. 30, 7 (2013).

16. Gaspari, M., Brighenti, F., D’Ercole, A. \& Melioli, C. AGN feedback in galaxy groups: the delicate touch of self-regulated outflows. Mon. Not. R. Astron. Soc. 415, 1549-1568 (2011).

17. Gaspari, M. \& Sadowski, A. Unifying the micro and macro properties of AGN feeding and feedback. Astrophys. J. 837, 149 (2017).

18. Krause, M. et al. A new connection between the jet opening angle and the large-scale morphology of extragalactic radio sources. Mon. Not. R. Astron. Soc. 427, 3196-3208 (2012)

19. Morganti, R., Killeen, N. E. B., Ekers, R. D. \& Oosterloo, T. A. Centaurus A: multiple outbursts or bursting bubble? Mon. Not. R. Astron. Soc. 307, 750-760 (1999).

20. Kraft, R. P. et al. The jet heated X-ray filament in the Centaurus A northern middle radio lobe. Astrophys. J. 698, 2036-2047 (2009).

21. Neff, S. G., Eilek, J. A. \& Owen, F. N. The complex north transition region of Centaurus A: a galactic wind. Astrophys. J. 802, 88 (2015).

22. Struve, C., Oosterloo, T. A., Morganti, R. \& Saripalli, L. Centaurus A: morphology and kinematics of the atomic hydrogen. Astron. Astrophys. 515, 67 (2010).

23. Junkes, N., Haynes, R. F., Harnett, J. I. \& Jauncey, D. L. Radio polarization surveys of Centaurus A (NGC 5128). I. The complete radio source at lambda $6.3 \mathrm{~cm}$. Astron. Astrophys. 269, 29-38 (1993).

24. Cooper, J. L., Bicknell, G. V., Sutherland, R. S. \& Bland-Hawthorn, J. Starburst-driven galactic winds: filament formation and emission processes. Astrophys. J. 703, 330-347 (2009).

25. Gronke, M. \& Oh, S. P. The growth and entrainment of cold gas in a hot wind. Mon. Not. R. Astron. Soc. 480, 111-115 (2018).

26. Santoro, F. et al. The outer filament of Centaurus A as seen by MUSE Astron. Astrophys. 575, 4 (2015).

27. Hamer, S. et al. MUSE discovers perpendicular arcs in the inner filament of Centaurus A. Astron. Astrophys. 575, 3 (2015).
28. Crockett, R. M. et al. Triggered star formation in the inner filament of Centaurus A. Mon. Not. R. Astron. Soc. 421, 1603-1623 (2012).

29. Crnojević, D. et al. The extended halo of Centaurus A: uncovering satellites, streams, and substructures. Astrophys. J. 823, 19 (2016).

30. Wang, J., Hammer, F., Rejkuba, M., Crnojević, D. \& Yang, Y. A recent major merger tale for the closest giant elliptical galaxy Centaurus A. Mon. Not. R. Astron. Soc. 498, 2766-2777 (2020).

31. Bowman, J. D. et al. Science with the Murchison Widefield Array. Publ. Astron. Soc. Aust. 30, 31 (2013).

32. Turner, W. et al. Technical Report SKA-TEL-SKO-0000008, SKA Phase 1 System Requirements Specification (SKA Organisation, 2016).

33. Wayth, R. B. et al. The Phase II Murchison Widefield Array: design overview. Publ. Astron. Soc. Aust. 35, 33 (2018).

34. Beardsley, A. P. et al. Science with the Murchison Widefield Array: Phase I results and Phase II opportunities. Publ. Astron. Soc. Aust. 36, 50 (2019).

35. Offringa, A. R. et al. Post-correlation radio frequency interference classification methods. Mon. Not. R. Astron. Soc. 405, 155-167 (2010).

36. Offringa, A. R., van de Gronde, J. J. \& Roerdink, J. B. T. M. A morphological algorithm for improving radio-frequency interference detection. Astron. Astrophys. 539, 95 (2012).

37. Offringa, A. R. et al. Parametrizing epoch of reionization foregrounds: a deep survey of low-frequency point-source spectra with the Murchison Widefield Array. Mon. Not. R. Astron. Soc. 458, 1057-1070 (2016).

38. Offringa, A. R. et al. WSCLEAN: an implementation of a fast, generic wide-field imager for radio astronomy. Mon. Not. R. Astron. Soc. 444, 606-619 (2014)

39. van der Tol, S., Veenboer, B. \& Offringa, A. R. Image domain gridding: a fast method for convolutional resampling of visibilities. Astron. Astrophys. 616, 27 (2018).

40. Sokolowski, M. et al. Calibration and stokes imaging with full embedded element primary beam model for the Murchison Widefield Array. Publ. Astron. Soc. Aust. 34, 62 (2017).

41. McKinley, B. et al. The jet/wind outflow in Centaurus A: a local laboratory for AGN feedback. Mon. Not. R. Astron. Soc. 474, 4056-4072 (2018).

42. Anderson, C. et al. The extraordinary linear polarisation structure of the southern Centaurus A lobe revealed by ASKAP. Galaxies 6, 127 (2018).

43. Veilleux, S. et al. MMTF: The Maryland-Magellan Tunable Filter. Astron. J. 139, 145-157 (2010).

44. Magnier, E. A. \& Cuillandre, J.-C. The Elixir system: data characterization and calibration at the Canada-France-Hawaii Telescope. Publ. Astron. Soc. Pac. 116, 449-464 (2004).

45. Gwyn, S. D. J. MegaPipe: the MegaCam image stacking pipeline at the Canadian Astronomical Data Centre. Publ. Astron. Soc. Pac. 120, 212 (2008).

46. Bertin, E. et al. The TERAPIX pipeline. In Astronomical Society of the Pacific Conference Proceedings, Astronomical Data Analysis Software and Systems XI Vol. 281 (eds Bohlender, D. A. et al.) 228-237 (Astronomical Society of the Pacific, 2002).

47. Bertin, E. \& Arnouts, S. SExtractor: Software for source extraction. Astron. Astrophys. Suppl. 117, 393-404 (1996).

48. Turner, R. J. \& Shabala, S. S. Energetics and lifetimes of local radio active galactic nuclei. Astrophys. J. 806, 59 (2015).

49. Eisenreich, M., Naab, T., Choi, E., Ostriker, J. P. \& Emsellem, E. Active galactic nuclei feedback, quiescence and circumgalactic medium metal enrichment in early-type galaxies. Mon. Not. R. Astron. Soc. 468, 751-768 (2017).

50. Yoon, D. et al. Active galactic nucleus feedback in an elliptical galaxy with the most updated AGN physics. II. High angular momentum case. Astrophys. J. 864, 6 (2018).

51. Li, Y.-P. et al. Stellar and AGN feedback in isolated early-type galaxies: the role in regulating star formation and ISM properties. Astrophys. J. 866, 70 (2018).

52. $\mathrm{Su}, \mathrm{K} .-\mathrm{Y}$. et al. Which AGN jets quench star formation in massive galaxies? Mon. Not. R. Astron. Soc. 507, 175-204 (2021).

53. Bondi, H. On spherically symmetrical accretion. Mon. Not. R. Astron. Soc 112, 195-204 (1952).

54. Gaspari, M. et al. The X-ray halo scaling relations of supermassive black holes. Astrophys. J. 884, 169 (2019).

55. Best, P. N. \& Heckman, T. On the fundamental dichotomy in the local radio-AGN population: accretion, evolution and host galaxy properties. Mon. Not. R. Astron. Soc. 421, 1569-1582 (2012).

56. Hardcastle, M. Interpreting radiative efficiency in radio-loud AGNs. Nat. Astron. 2, 273-274 (2018).

57. Gaspari, M. The self-regulated AGN feedback loop: the role of chaotic cold accretion. Proc. Int. Astron. Union 11, 17-20 (2016).

58. Gaspari, M. Shaken snow globes: kinematic tracers of the multiphase condensation cascade in massive galaxies, groups, and clusters. Astrophys. J. 854, 167 (2018)

59. Gaspari, M., Temi, P. \& Brighenti, F. Raining on black holes and massive galaxies: the top-down multiphase condensation model. Mon. Not. R. Astron. Soc. 466, 677-704 (2017). 
60. Gaspari, M. Shaping the X-ray spectrum of galaxy clusters with AGN feedback and turbulence. Mon. Not. R. Astron. Soc. 451, 60-64 (2015).

61. Gaspari, M., Melioli, C., Brighenti, F. \& D’Ercole, A. The dance of heating and cooling in galaxy clusters: three-dimensional simulations of self-regulated active galactic nuclei outflows. Mon. Not. R. Astron. Soc. 411, 349-372 (2011).

62. Kraft, R. P. et al. X-ray emission from the hot interstellar medium and southwest radio lobe of the nearby radio galaxy Centaurus A. Astrophys. J. 592, 129-146 (2003).

63. Heywood, I. et al. Inflation of 430-parsec bipolar radio bubbles in the Galactic Centre by an energetic event. Nature 573, 235-237 (2019).

64. Su, M., Slatyer, T. R. \& Finkbeiner, D. P. Giant gamma-ray bubbles from Fermi-LAT: active galactic nucleus activity or bipolar galactic wind? Astron. J. 724, 1044-1082 (2010)

65. Dobler, G. et al. The Fermi haze: A gamma-ray counterpart to the microwave haze. Astron. J. 717, 825-842 (2010).

66. Carretti, E. et al. Giant magnetized outflows from the centre of the Milky Way. Nature 493, 66-69 (2013).

67. Finkbeiner, D. P. Microwave interstellar medium emission observed by the Wilkinson Microwave Anisotropy Probe. Astrophys. J. 614, 186-193 (2004).

68. Crocker, R. M., Bicknell, G. V., Taylor, A. M. \& Carretti, E. A unified mode of the Fermi bubbles, microwave haze, and polarized radio lobes: reverse shocks in the Galactic Center's giant outflows. Astrophys. J. 808, 107 (2015).

69. Merloni, A., Nandra, K. \& Predehl, P. eROSITAa's X-ray eyes on the Universe. Nat. Astron. 4, 634-636 (2020).

70. Predehl, P. et al. Detection of large-scale X-ray bubbles in the Milky Way halo. Nature 588, 227-231 (2020).

71. Bogdán, Á. \& Gilfanov, M. Soft band X/K luminosity ratios for gas-poor early-type galaxies. Astron. Astrophys. 512, 16 (2010).

72. Sądowski, A. \& Gaspari, M. Kinetic and radiative power from optically thin accretion flows. Mon. Not. R. Astron. Soc. 468, 1398-1404 (2017).

73. Gaspari, M., Brighenti, F. \& Temi, P. Mechanical AGN feedback: controlling the thermodynamical evolution of elliptical galaxies. Mon. Not. R. Astron. Soc. 424, 190-209 (2012).

74. Godfrey, L. E. H. \& Shabala, S. S. Mutual distance dependence drives the observed jet-power-radio-luminosity scaling relations in radio galaxies. Mon. Not. R. Astron. Soc. 456, 1172-1184 (2016).

75. Eilek, J. A. The dynamic age of Centaurus A. New J. Phys. 16, 045001 (2014).

76. Wykes, S. et al. Mass entrainment and turbulence-driven acceleration of ultra-high energy cosmic rays in Centaurus A. Astron. Astrophys. 558, 19 (2013).

77. O'Sullivan, S. P. et al. Thermal plasma in the giant lobes of the radio galaxy Centaurus A. Astrophys. J. 764, 162 (2013).

78. Stawarz, Ł. et al. Giant lobes of Centaurus A radio galaxy observed with the Suzaku X-ray satellite. Astrophys. J. 766, 48 (2013).

79. Schiminovich, D., van Gorkom, J. H., van der Hulst, J. M. \& Kasow, S. Discovery of neutral hydrogen associated with the diffuse shells of NGC 5128 (Centaurus A). Astrophys. J. Lett. 423, 101 (1994).

80. Graham, J. A. \& Price, R. M. The gaseous filaments in the northeast halo region of NGC 5128 (Centaurus A). Astrophys. J. 247, 813-822 (1981).

81. Morganti, R. et al. The nature of the optical filaments in Centaurus A. Evidence for a beamed ionizing continuum. Mon. Not. R. Astron. Soc. 249, 91-112 (1991).

82. Santoro, F. et al. The jet-ISM interaction in the outer filament of Centaurus A. Astron. Astrophys. 574, 89 (2015).

83. Sutherland, R. S., Bicknell, G. V. \& Dopita, M. A. Shock excitation of the emission-line filaments in Centaurus A. Astrophys. J. 414, 510 (1993).

84. Gaspari, M., Ruszkowski, M. \& Sharma, P. Cause and effect of feedback: multiphase gas in cluster cores heated by AGN jets. Astrophys. J. 746, 94 (2012).

85. Kraft, R. P. et al. Evidence for nonhydrostatic gas motions in the hot interstellar medium of Centaurus A. Astrophys. J. 677, 97 (2008).

86. Król, D. Ł., Marchenko, V., Ostrowski, M. \& Stawarz, Ł. An analysis of soft $\mathrm{X}$-ray structures at kiloparsec distances from the active nucleus of Centaurus A galaxy. Astrophys. J. 903, 107 (2020).

87. Israel, F. P. et al. The outflow of gas from the Centaurus A circumnuclear disk. Atomic spectral line maps from Herschel/PACS and APEX. Astron. Astrophys. 599, 53 (2017).

88. Veilleux, S., Cecil, G. \& Bland-Hawthorn, J. Galactic winds. Annu. Rev. Astron. Astrophys. 43, 769-826 (2005).

89. DeBoer, D. R. et al. Australian SKA Pathfinder: a high-dynamic range wide-field of view survey telescope. Proc. IEEE 97, 1507-1521 (2009).

90. Hotan, A. W. et al. The Australian Square Kilometre Array Pathfinder: system architecture and specifications of the Boolardy engineering test array. Publ. Astron. Soc. Aust. 31, 41 (2014).

91. Camilo, F. et al. Revival of the magnetar PSR J1622-4950: observations with MeerKAT, Parkes, XMM-Newton, Swift, Chandra, and NuSTAR. Astron. J. 856, 180 (2018).
92. Jonas, J. et al. The MeerKAT radio telescope. In Proc. of MeerKAT Science: On the Pathway to the SKA Proceedings of Science, Vol. 277 (eds Taylor, R. et al.) Article ID 001 (SISSA, 2018).

93. Tingay, S. J., Preston, R. A. \& Jauncey, D. L. The subparsec-scale structure and evolution of Centaurus A. II. Continued very long baseline array monitoring. Astron. J. 122, 1697-1706 (2001).

94. Jones, D. L. et al. Discovery of a sub-parsec radio counterjet in the nucleus of Centaurus A. Astrophys. J. 466, 63 (1996).

95. Tombesi, F. et al. Ultrafast outflows in radio-loud active galactic nuclei. Mon. Not. R. Astron. Soc. 443, 2154-2182 (2014)

96. Nesvadba, N. P. H. et al. Gas kinematics in powerful radio galaxies at $z \sim 2$ energy supply from star formation, AGN, and radio jets. Astron. Astrophys. 600, 121 (2017).

97. Morganti, R. Radio jets clearing the way through galaxies: the view from HI and molecular gas. Proc. Int. Astron. Union 313, 283-288 (2015).

98. Bicknell, G. V. et al. AGN feedback by relativistic jets. Proc. Int. Astron Union 313, 101-107 (2015).

99. van Gorkom, J. H. et al. H i absorption in radio elliptical galaxiesevidence for infall. Astron. J. 97, 708-719 (1989).

100. van der Hulst, J. M. et al. The H i absorption in NGC 5128 (Centaurus A). Astrophys. J. 264, 37-41 (1983).

101. Best, P. N. The host galaxies of radio-loud active galactic nuclei: mass dependences, gas cooling and active galactic nuclei feedback. Mon. Not. R. Astron. Soc. 362, 25-40 (2005).

102. Neumayer, N. The supermassive black hole at the heart of Centaurus A revealed by the kinematics of gas and stars. Publ. Astron. Soc. Aust. 27, 449-456 (2010).

103. Cooper, B. F. C., Price, R. M. \& Cole, D. J. A study of the decimetric emission and polarization of Centaurus A. Aust. J. Phys. 18, 589-626 (1965).

104. McNamara, B. R. et al. An energetic AGN outburst powered by a rapidly spinning supermassive black hole or an accreting ultramassive black hole. Astrophys. J. 698, 594-605 (2009).

105. McDonald, M. et al. Observational evidence for enhanced black hole accretion in giant elliptical galaxies. Astrophys. J. 908, 85 (2021).

106. Donnari, M. et al. The star formation activity of IllustrisTNG galaxies: main sequence, UVJ diagram, quenched fractions, and systematics. Mon. Not. R. Astron. Soc. 485, 4817-4840 (2019).

107. Zinger, E. et al. Ejective and preventative: the IllustrisTNG black hole feedback and its effects on the thermodynamics of the gas within and around galaxies. Mon. Not. R. Astron. Soc. 499, 768-792 (2020).

108. Bassini, L. et al. Black hole mass of central galaxies and cluster mass correlation in cosmological hydro-dynamical simulations. Astron. Astrophys. 630, 144 (2019).

109. Truong, N., Pillepich, A. \& Werner, N. Correlations between supermassive black holes and hot gas atmospheres in IllustrisTNG and X-ray observations. Mon. Not. R. Astron. Soc. 501, 2210-2230 (2021).

110. Mitchell, P. D. et al. Galactic outflow rates in the EAGLE simulations. Mon. Not. R. Astron. Soc. 494, 3971-3997 (2020).

111. Davé, R. et al. SIMBA: cosmological simulations with black hole growth and feedback. Mon. Not. R. Astron. Soc. 486, 2827-2849 (2019).

112. Beckmann, R. S. et al. Cosmic evolution of stellar quenching by AGN feedback: clues from the Horizon-AGN simulation. Mon. Not. R. Astron. Soc. 472, 949-965 (2017).

113. Tremmel, M. et al. Introducing ROMULUSC: a cosmological simulation of a galaxy cluster with an unprecedented resolution. Mon. Not. R. Astron. Soc. 483, 3336-3362 (2019).

114. Marinacci, F. et al. First results from the IllustrisTNG simulations: radio haloes and magnetic fields. Mon. Not. R. Astron. Soc. 480, 5113-5139 (2018).

115. Naiman, J. P. et al. First results from the IllustrisTNG simulations: a tale of two elements-chemical evolution of magnesium and europium. Mon. Not. R. Astron. Soc. 477, 1206-1224 (2018).

116. Nelson, D. et al. First results from the IllustrisTNG simulations: the galaxy colour bimodality. Mon. Not. R. Astron. Soc. 475, 624-647 (2018)

117. Pillepich, A. et al. First results from the IllustrisTNG simulations: the stellar mass content of groups and clusters of galaxies. Mon. Not. R. Astron. Soc. 475, 648-675 (2018).

118. Springel, V. et al. First results from the IllustrisTNG simulations: matter and galaxy clustering. Mon. Not. R. Astron. Soc. 475, 676-698 (2018).

119. Pillepich, A. et al. First results from the TNG50 simulation: the evolution of stellar and gaseous discs across cosmic time. Mon. Not. R. Astron. Soc. 490, 3196-3233 (2019).

120. Brennan, R. et al. Momentum-driven winds from radiatively efficient black hole accretion and their impact on galaxies. Astrophys. J. 860, 14 (2018).

121. Gofford, J. et al. The Suzaku view of highly ionized outflows in AGN-I. Statistical detection and global absorber properties. Mon. Not. R. Astron. Soc. 430, 60-80 (2013). 
122. Gofford, J. et al. The Suzaku view of highly ionized outflows in AGN-II. Location, energetics and scalings with bolometric luminosity. Mon. Not. R. Astron. Soc. 451, 4169-4182 (2015).

123. Tombesi, F. et al. Feeding and feedback in the powerful radio galaxy $3 \mathrm{C}$ 120. Astrophys. J. 838, 16 (2017).

124. Harrison, C. M. et al. The KMOS AGN survey at high redshift (KASHz): the prevalence and drivers of ionized outflows in the host galaxies of X-ray AGN. Mon. Not. R. Astron. Soc. 456, 1195-1220 (2016).

125. Schmidt, M., Schneider, D. P. \& Gunn, J. E. Spectroscopic CCD surveys for quasars at large redshift IV: evolution of the luminosity function from quasars detected by their Lyman-alpha emission. Astron. J. 110, 68-77 (1995).

126. Leung, G. C. K. et al. The MOSDEF survey: the prevalence and properties of galaxy-wide AGN-driven outflows at $z \sim 2$. Astrophys. J. 849, 48 (2017).

127. Circosta, C. et al. SUPER I: toward an unbiased study of ionized outflows in $z \sim 2$ active galactic nuclei: survey overview and sample characterization. Astron. Astrophys. 620, 82 (2018).

128. Kakkad, D. et al. SUPER II: spatially resolved ionised gas kinematics and scaling relations in $z \sim 2$ AGN host galaxies. Astron. Astrophys. 642, 147 (2020).

129. Bischetti, M. et al. Widespread QSO-driven outflows in the early Universe. Astron. Astrophys. 630, 59 (2019).

130. Croston, J. H. et al. High-energy particle acceleration at the radio-lobe shock of Centaurus A. Mon. Not. R. Astron. Soc. 395, 1999-2012 (2009).

131. Keel, W. C. et al. Optical detection of star formation in a cold dust cloud in the counterjet direction of Centaurus A. Astron. J. 157, 66 (2019).

132. O'Sullivan, E., Ponman, T. J. \& Collins, R. S. X-ray scaling properties of early-type galaxies. Mon. Not. R. Astron. Soc. 340, 1375-1399 (2003).

\section{Acknowledgements}

This scientific work makes use of the Murchison Radio-Astronomy Observatory, operated by the Commonwealth Scientific and Industrial Research Organisation. We acknowledge the Wajarri Yamatji people as the traditional owners of the Observatory site. Support for the operation of the MWA is provided by the Australian Government through the National Collaborative Research Infrastructure Strategy, under a contract to Curtin University administered by Astronomy Australia Limited. We acknowledge the Pawsey Supercomputing Centre, which is supported by the Western Australian and Australian Governments. M.G. acknowledges partial support by National Aeronautics and Space Administration Chandra GO8-19104X/GO9-20114X and Hubble Space Telescope GO-15890.020/023-A, and the BlackHoleWeather programme. M.S.C. and M.M. thank S. Courteau and D. Simons for CFHT Director's discretionary time via the Mauna Kea Graduate School programme. Research by D.C. is supported by National Science Foundation grant AST-1814208. This work was supported by an Australian Research Council Future Fellowship under grant FT180100321. The authors thank A. Pillepich, R. Morganti, R. Wahl-Olsen and M. Sidonio for useful discussions and guidance during preparation of the manuscript.

\section{Author contributions}

Processing of the radio data was performed by B.M. A.R.O. contributed software development crucial to the success of the radio-data processing. Processing of the lower resolution $\mathrm{H} \alpha$ data in Fig. 2 and visualization of the radio data in Extended Data Fig. 1 was performed by C.M. Processing of the CFHT data was performed by M.S.C., M.M. and S.D.J.G. R.P.K. provided the X-ray data and input regarding its role in the analysis. D.C. provided the RGB star map and input regarding the merger history of Centaurus A.M.G., S.S.S., S.V., S.J.T. and J.B-H. provided input on modelling, theoretical aspects and consistency with observations. B.M.G. and M.J-H. played critical roles in the upgrade of the MWA that enabled the radio observations. All authors contributed to the interpretation of the data and the discussion presented, and all were involved in the writing of the paper.

\section{Competing interests}

The authors declare no competing interests.

\section{Additional information}

Extended data is available for this paper at https://doi.org/10.1038/s41550-021-01553-3. Correspondence and requests for materials should be addressed to B. McKinley.

Peer review information Nature Astronomy thanks Martin Hardcastle, Gene Leung and the other, anonymous, reviewer(s) for their contribution to the peer review of this work.

Reprints and permissions information is available at www.nature.com/reprints.

Publisher's note Springer Nature remains neutral with regard to jurisdictional claims in published maps and institutional affiliations.

(c) The Author(s), under exclusive licence to Springer Nature Limited 2022 

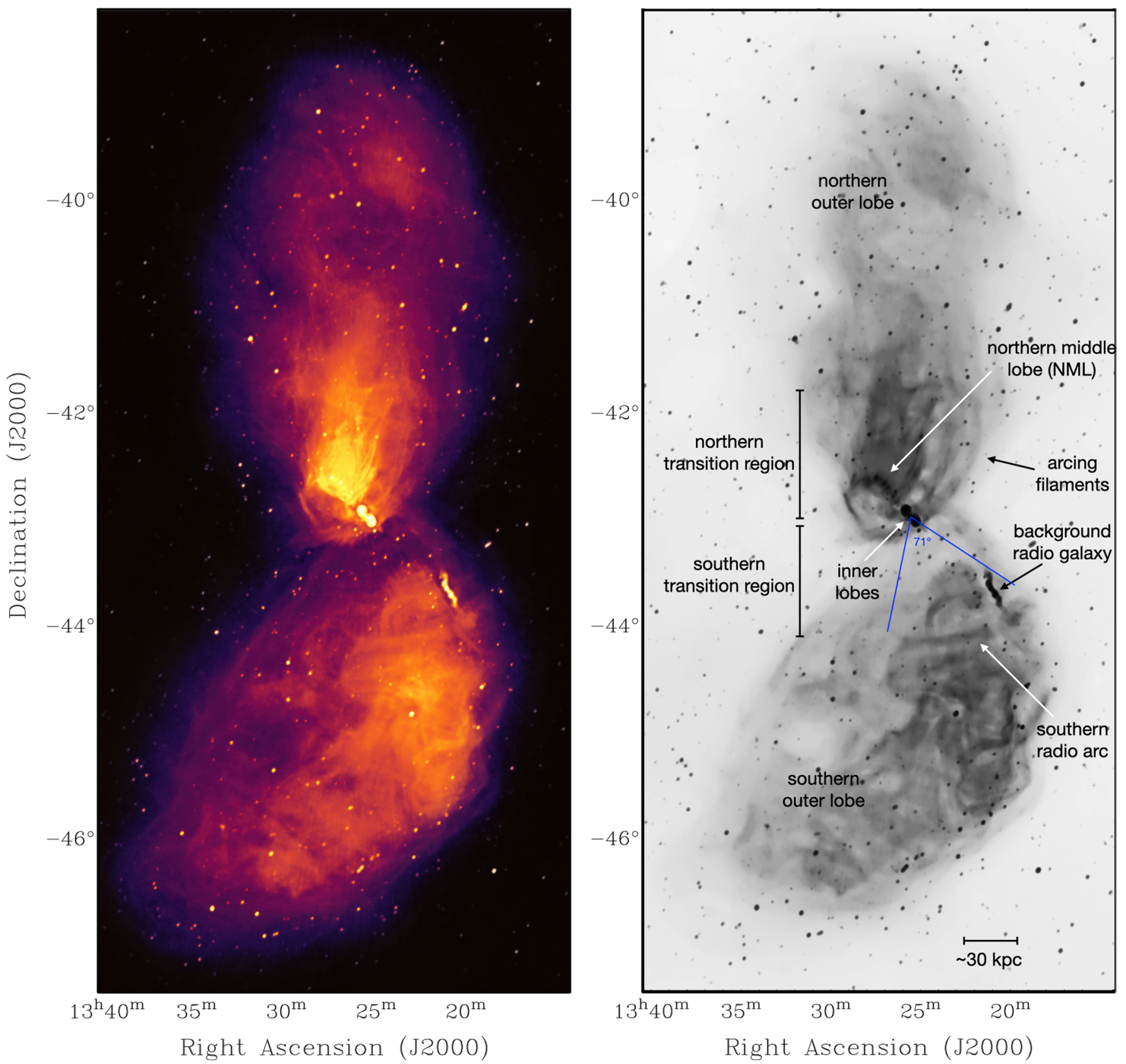

Extended Data Fig. 1 | Centaurus A with modified intensity and color scale. Centaurus A as imaged with the MWA at $185 \mathrm{MHz}$, with the intensity scale manipulated in order to deal with the extreme dynamic range of the image. The intensity scale has been altered in a non-linear fashion, so the greyscale/ colorscale range is not meaningful and is not displayed. This is intended as a qualitative representation of the data only. The average restoring beam is a Gaussian of width $1.5 \times 1.2$ arcmin with a major axis position angle of $155^{\circ}$. Right: The image has been stretched using the astrophotography program PixInsight (see Methods) to effectively identify and label the key features of the radio galaxy. This image was not used for quantitative analyses, but is used here to indicate the positions of various features discussed in the text, including the $71^{\circ}$ opening angle estimated for the southern component of the broad AGN outflow (blue lines). Left: Color has been added to the stretched image in the right panel using Photoshop, in order to better show the large-scale diffuse emission along with the finer details of the radio lobes. Yellow and white show higher intensities, while red and magenta show lower intensities. 


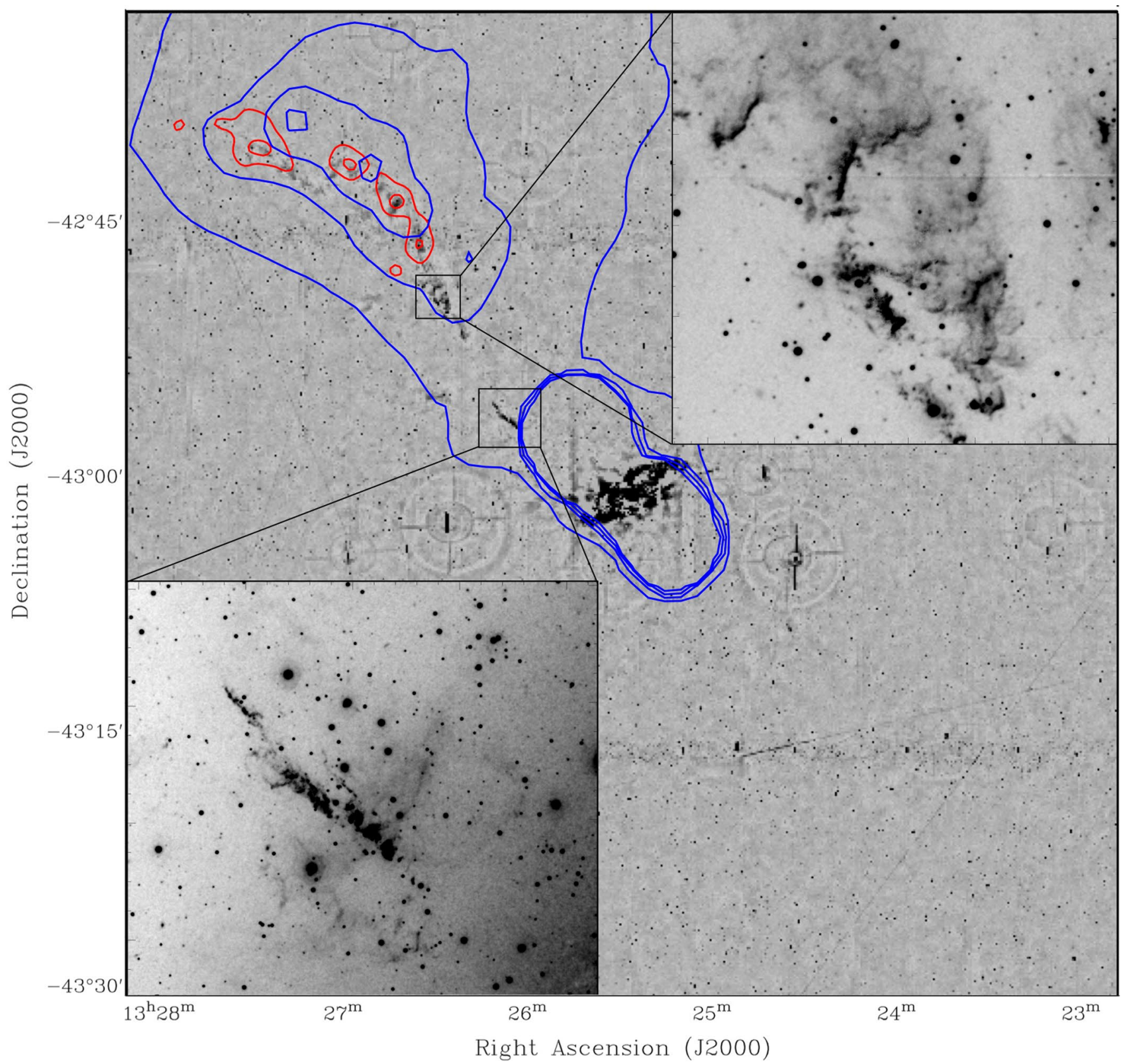

Extended Data Fig. 2 | Centaurus A in H $\alpha$ emission with radio and X-ray contours. Widefield $\mathrm{H} \alpha$ image of the central $1^{\circ}$ of Centaurus A taken with CFHT. The point spread function is characterised by a two-dimensional Moffat function with a core width of 5.7 arcsec (see Methods). Red contours show the $X$-ray knots from Fig. 2 at approximately $3 \sigma$ and $6 \sigma$ above the background noise, where $\sigma$ is the standard deviation of a group of background pixels. Blue contours are from the MWA radio image at $0.5,1,1.5,2 \mathrm{Jy} /$ beam. The average restoring beam of the MWA image from which the blue contours were obtained is a Gaussian of width $1.5 \times 1.2$ arcmin with a major axis position angle of $155^{\circ}$. Top right inset: higher resolution ( 0.8 arcsec) MMTF image ${ }^{41}$ zoom in on part of the outer filament showing the arcs of ionised gas which resemble bow shocks. Bottom left inset: higher resolution ( 0.8 arcsec) MMTF image $^{41}$ zoom in on inner filament showing a similarly shaped arc of ionised gas, indicating the presence of a wind from the central galaxy. 